\title{
La tendencia del factor de cresta ayuda a detectar eventos nacientes; circuito electrónico, programas y aplicaciones a señales de diversos campos
}

\section{The Tendency of the Crest Factor Helps Detect Nascent Events; Electronic Circuit, Software and Applications to Signals from Diverse Fields}

\author{
Núñez-Pérez Ricardo Francisco \\ Electrónica y Telecomunicaciones \\ Centro de Investigación Científica y de Educación Superior de Ensenada, CICESE \\ Correo:rnunez@cicese.mx
}

Información del artículo: recibido: abril de 2012, aceptado: marzo de 2013

\section{Resumen}

Dentro de las técnicas de análisis de señales en el dominio del tiempo, el factor de cresta (FC) es sin duda una de las más simples y rápidas de implementar por medio de circuitos electrónicos o programas de cómputo. Es por eso que se han utilizado confiablemente para cuidar maquinaria y evaluar la calidad de la alimentación eléctrica. Uno de los fabricantes importantes de instrumentos para estos fines es Bruel y Kjaer y define al factor de cresta de una señal de voltaje o corriente repetitiva como el cociente entre el nivel del pico máximo y su valor eficaz durante un tiempo determinado. En este trabajo, se trata de averiguar experimentalmente el potencial del FC y de su tendencia para detectar el nacimiento y evolución de eventos en varios campos del conocimiento; ya sea generándolo por medio de un circuito electrónico que se desarrolla o calculándolo por medio de rutinas que se realizan con los programas DADISP y LabVIEW. Se validan y corroboran los resultados de todos los factores mencionados y de sus tendencias a través de un estudio comparativo entre ellos y las características y especificaciones planteadas. Los resultados fueron aceptables, de tal suerte que las herramientas se aplican para detectar averías tempranas en máquinas eléctricas, identifican diferencias entre la caosidad de circuitos con esas dinámicas, detectan deficiencias respiratorias o estertores anormales en pacientes y detectan distorsiones perjudiciales en la corriente eléctrica; todo esto con base en simu-

\section{Descriptores:}

- factor de cresta

- tendencia

- circuitos caóticos

- cuidado de maquinaria

- distorsión eléctrica 
laciones y mediciones realizadas para cada uno de los 4 casos estudiados. Otras aplicaciones originales del FC que se proponen son: a) controlar el caos en circuitos electrónicos que agitan/mezclan procesos industriales y b) corregir el factor de potencia de cargas no-lineales e inductivas. Se contempla a mediano plazo estudiar y utilizar un FC que considere la señal máxima de pico a pico; se piensa que así podría mejorarse su detección de eventos.

\begin{abstract}
Within the signal analysis techniques in the time domain, the crest factor $(C F)$ is undoubtedly one of the most simple and fast to implement using electronic circuits and/or software. That's why it has been used reliably to care for machinery and to evaluate the quality of supply. One of the major manufacturers of instruments for these purposes is Bruel and Kjaer and defines the crest factor of voltage or repetitive current signal as the ratio of the peak level and its rms value during a certain period of time. In this paper, we try to find out experimentally the potential of CF and their tendency to detect the nascent and evolution of events in various fields of knowledge, either by generating it with a developed electronic circuit, or with calculations, through routines that are performed with the programs DADISP and LabVIEW. The results are validated and checked for all the above factors and trends through a comparison between them and the proposed features and specifications. The results were acceptable so that the tools were applied to detect early faults in electrical machines, to identify chaosity differences between the circuits with these dynamics, to detect abnormal respiratory distress or rales in patients and to detect harmful distortions in the electrical current, all this based on simulations and measurements for each of the 4 cases studied. Other CF original applications proposed are: a) control of chaos in electronic circuits that stir/ mix industrial processes and b) correct the power factor of non-linear and inductive loads. A medium-term study and use a CF that considers the maximum signal peak to peak is contemplated, and it is thought that it can improve event detection.
\end{abstract}

\section{Introducción}

Dentro de las técnicas de análisis de señales en el dominio del tiempo, el factor de cresta FC (Fink y Beaty, 1990; Bruel y Kjaer, 1989a y b) es sin duda una de las más simples y rápidas de implementar por medio de circuitos electrónicos o programas de cómputo. Prueba de ello, es que se han utilizado confiablemente en programas para el cuidado de maquinaria, -ya que miden la vibración mecánica y otras variables- y para evaluar la calidad de la alimentación eléctrica -basados en la distorsión de la corriente en la carga (Fluke, 1990; Cáceres, 2008)-implantados por compañías que desarrollan e integran instrumentos y equipos para esta labor (Bruel y Kjaer, 1989a y b; CSI, 1988), todo esto desde hace ya varios años. En particular, se ha visto que este factor resulta apropiado para la detección temprana del deterioro en rodamientos, rotores y cajas de engranes (Bruel y Kjaer, 1989b; Núñez, 1987; Félix, 1992). Esto se debe a que las señales en juego son una mezcla de pe- riódicas, pulsantes periódicas, aperiódicas, entre otras. Por ello, cuando las sintomáticas pulsantes se incrementan distorsionan aún más a las periódicas y por lo tanto el FC del conjunto crece, y cuando la magnitud de la frecuencia fundamental aumenta, con respecto al resto de las señales, el factor mencionado disminuye; estas variaciones en el factor mencionado revisten un potencial interesante de aplicación. Aunque en ese sentido, se conocen contadas aplicaciones de este factor en otros campos del conocimiento, por lo que en este trabajo se propone utilizarlo en el de los circuitos con dinámica caótica y en el de la biomedicina del sistema respiratorio, como se señalará más adelante.

Uno de los fabricantes más importantes en estos instrumentos para el cuidado de maquinaria, Bruel y Kjaer (1985), define el factor de cresta de una señal de voltaje o corriente repetitiva como el cociente entre el nivel del pico máximo y su valor eficaz durante un tiempo predeterminado (Fink y Beaty, 1990; Lancaster, 1998; Cáceres, 2008). Como se ve, el factor es adimensional y se 
acostumbra graficar su tendencia; si aumenta o disminuye y con qué tasa lo hace, ésta es la mejor manera de saber que algo imprevisto está sucediendo con las señales. Esta acción facilita la identificación temprana de algún síntoma e indica cuándo es necesario utilizar instrumentos de diagnóstico más sofisticados, como los del dominio de la frecuencia. Algunas veces es conveniente tomar las medidas apropiadas, ya que el factor puede alterarse debido a la incorporación de ruido eléctrico en la señal de información.

En este trabajo, se trata de comprobar experimentalmente el potencial del FC y de su tendencia en la detección de eventos nacientes tanto por medio de un circuito electrónico sencillo que lo genera, como por los programas DADISP y LabVIEW, que lo calculan para validar resultados y poder aplicar confiablemente ambos procedimientos en la detección temprana de rodamientos dañados y rotores desbalanceados (Bruel y Kjaer, 1989a) y de distorsión en la corriente de la red eléctrica (Cáceres, 2008; Fink y Beaty, 1990; Early et al., 1989) en los campos del mantenimiento de maquinaria industrial y del consumo eléctrico eficiente, respectivamente. Así como también, con base en la experiencia adquirida, aplicar los mismos procedimientos en los campos de la medición de la dinámica de los circuitos caóticos (Núñez, 2008) con fines de aplicación industrial y en la detección de síntomas tempranos de deficiencias respiratorias comunes (Charbonneau et al., 2000; Díaz, 2008; Quezada, 2011) para un posible monitoreo permanente de pacientes.

En la siguiente sección de explicación del FC, se define y describe el FC. Se presenta un circuito electrónico (Coughlin y Driscoll, 1982; Counts et al., 1982; National Semiconductor, 1984; Kitchin y Counts, 1983; Stout y Kaufman, 1973; Analog Devices, 1983, 1985) para obtenerlo y se describe el cálculo de este factor por medio de los programas DADISP (2002) y LabVIEW (National Instruments, 2002); se validan y corroboran los resultados de los factores mediante un estudio comparativo. En la sección aplicaciones se propone la aplicación de los procedimientos validados para obtener y calcular el FC y su tendencia de diversas señales para detectar: el nacimiento de averías mecánicas, el grado de caosidad en circuitos caóticos, la detección de deficiencias básicas o estertores del sistema respiratorio y el grado de distorsión de la corriente eléctrica impuesta por cargas no-lineales. En la sección de análisis se examinan y reportan los resultados más relevantes, se plantean las recomendaciones para la aplicación eficiente y novedosa de este procedimiento de análisis temporal de señales en el control de caos, el monitoreo continuo y en tiempo real de deficiencias respiratorias, la corrección del factor de potencia, entre otras.

En las conclusiones se menciona que el FC y su tendencia es un procedimiento confiable que cumple con las características y especificaciones planteadas y facilita la decisión de pasar a un análisis y diagnóstico con equipo más especializado. Se plantea estudiar el factor mencionado considerando ahora la diferencia entre los picos máximo positivo y negativo, además de buscar aplicaciones en otros campos del conocimiento. Finalmente se presentan los agradecimientos y las referencias.

\section{El factor de cresta; circuito electrónico, programas y validación}

Bruel y Kjaer (1989a y b, 1985) definen el factor de cresta, FC, de una señal de voltaje o corriente repetitiva como el cociente entre el nivel del pico máximo y su valor eficaz durante un tiempo predeterminado (definición 1) (Fink y Beaty, 1990; Lancaster, 1998; Cáceres, 2008). Para mostrar lo anterior, en la figura 1 se presenta una señal caótica de voltaje, de la cual se observan sus características básicas: nivel del pico máximo positivo y negativo, el valor eficaz calculado para el tiempo de evaluación, así como su media. La señal mencionada proviene del circuito de Lorenz (Lorenz, 1963; Márquez y Álvarez, 1996 y Núñez, 2006) y fue adquirida y posanalizada con el programa DADISP (2002) (Núñez, 1998). A partir de las características mencionadas y utilizando la definición 1, se obtienen los FC correspondientes que indican la relación que existen entre cada nivel de pico máximo y el valor eficaz. Este factor es adimensional pero para fines prácticos se manifiesta como un voltaje de c.d. y se estila graficar en el tiempo para poder observar su tendencia, es decir, si aumenta o disminuye y con qué tasa lo hace indica que algo imprevisto está sucediendo entre las señales, lo que facilita el diagnóstico temprano de algún síntoma y manifiesta que sí es necesario utilizar instrumentos de diagnóstico más especializado, como los que se utilizan en el dominio de la frecuencia (Félix, 1992).

El FC se define como:

$$
\begin{gathered}
\mathrm{FC}=\frac{\mathrm{Vp}}{\mathrm{Vef}} \text { para } 0<t \leq t_{0} \\
\mathrm{Vef}=\sqrt{\frac{1}{T} \int\left(\operatorname{Ve}(t)^{*} \operatorname{Ve}(t)\right) d t}
\end{gathered}
$$

donde:

$t_{0} \quad=$ tiempo de evaluación (segundos) 
$T \quad=$ periodo de evaluación (segundos)

$\operatorname{Ve}(t)=$ voltaje de la señal de entrada en c.a. (voltios)

$\mathrm{Vp}$ = voltaje del pico máximo de la señal en c.d. (voltios)

Vef $=$ voltaje del valor eficaz de la señal en c.d. (voltios)

Aunque la obtención del factor mencionado es sencilla y rápida, en algunos casos es necesario tener cuidado, ya que puede alterarse producto del ruido eléctrico que se incorpora a las señales periódicas de información.

En la figura 2 se presenta una descripción gráfica del cálculo del FC (explicado en el segundo punto de esta sección) de tres señales ideales generadas y analizadas con el programa DADISP (2002). Las señales son: cosenoidal (W1: gcos $(100,1 / 100,10))$, cuadrada (W5: gsqwave $(100,1 / 100,10))$, cosenoidal rectificada de media onda (W7) y sus FC obtenidos son: 1.4142 (W2), 1.0000 (W6) y 2.0000 (W8), respectivamente. Estos resultados coinciden plenamente con lo reportado en la bibliografía (Beckman Industrial, 1984). En las ventanas W3 y W4, se describe la evolución del voltaje de valor eficaz Vef de la cosenoidal y el traslape de éste (gráfica inferior) con la propia señal cosenoidal y con la evolución del FC (gráfica superior), respectivamente. Todas las señales generadas son de 1 voltio de pico, de $10 \mathrm{~Hz}$, con una trama de 100 muestras y muestreadas a $100 \mathrm{~Hz}$. Una señal cosenoidal ideal que se deforma presenta un FC mayor a 1.4142, dependiendo del grado de distorsión impuesto.

Obtención del FC por medio del circuito electrónico CFC

El circuito que obtiene el FC, llamado CFC, realiza las operaciones analógicas mostradas en el diagrama a cuadros de la figura 3 para generar la ecuación (1).

El CFC es sencillo, contiene tres etapas con operadores analógicos que se combinan para generar el nivel de voltaje de salida Vs representante del factor mencionado (1). Con fines de prueba, la señal de voltaje de entrada $\mathrm{Ve}(t)$ proviene de un generador de funciones Agilent 33120A (Agilent Technologies, 2000) y se trabaja en paralelo por los operadores respectivos para obtener el Vef y el nivel de voltaje de pico máximo positivo $\mathrm{Vp}$ al mismo tiempo y que, el operador divisor, haga lo propio para generar el voltaje de salida Vs. Los circuitos se diseñan siguiendo los criterios propuestos por Kitchin y Counts (1983); Stout y Kaufman (1976); Coughlin y Driscoll (1982) y Analog Devices (1985).

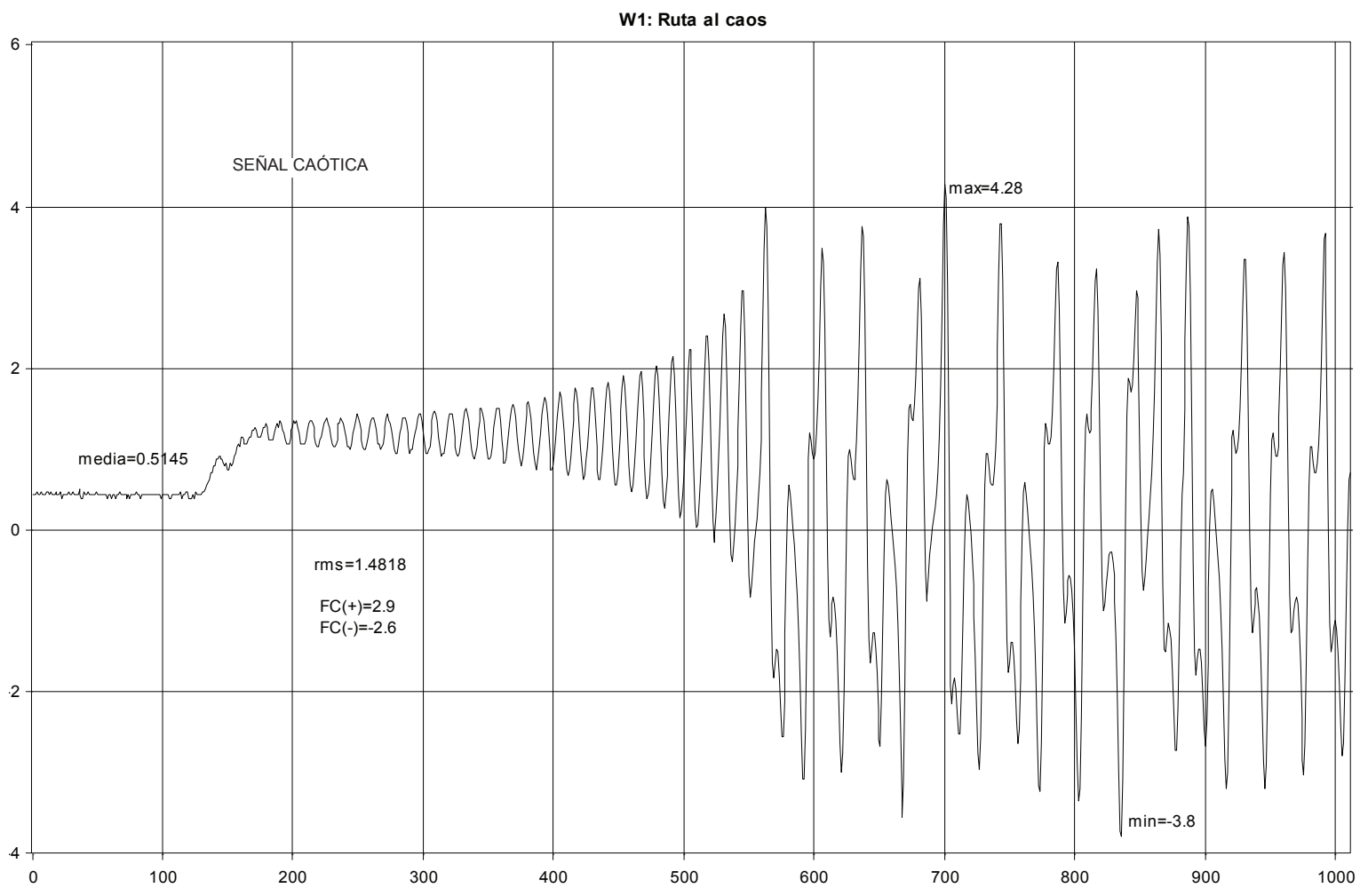

Figura 1. W1: señal caótica (Núñez, 2006) mostrando algunas de sus características: media, valor pico max/min, valor eficaz y sus FC. Se observa el inicio del caos a partir de los $570 \mathrm{~m} / \mathrm{seg}$ producto de la bifurcación (vertical: voltios, horizontal: m/seg) 

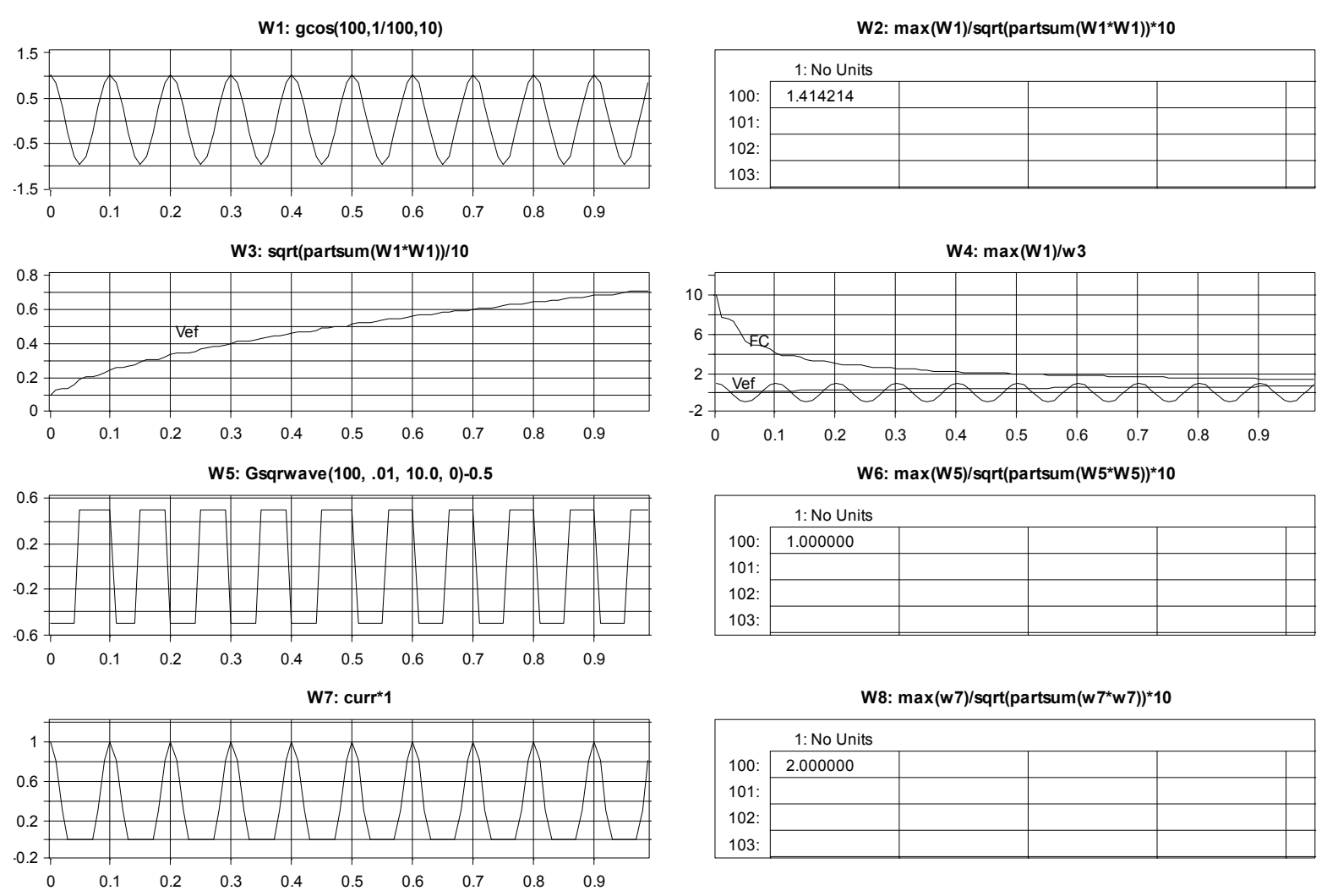

Figura 2. Señales ideales (W1: cosenoidal, W5: cuadrada y W7: cosenoidal rectificada de media onda) y sus correspondientes FC, generados y calculados con el programa DADISP, respectivamente. En W4 se muestra la relación entre Vef, Vp y el FC para W1 (vertical: voltios y horizontal: segundos)

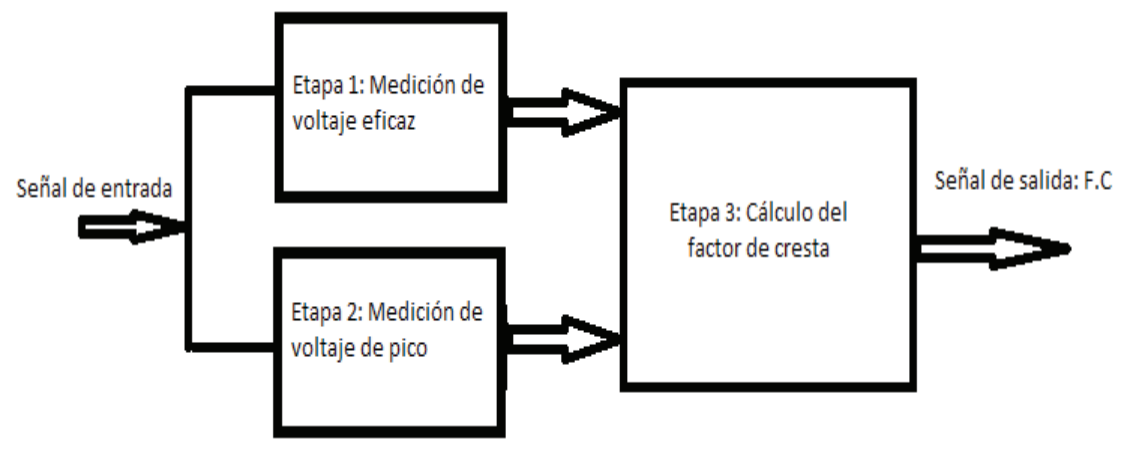

Figura 3. Diagrama cuadros del circuito electrónico CFC para obtener el FC liza el operador analógico AD536AJH de Analog Devices (1982, 1983) y se diseña e implementa atendiendo las características y especificaciones propuestas, siguiendo las recomendaciones del fabricante (Analog Devices, 1983) y de otros (Counts et al., 1982; Kitchin y Counts, 1983 y Núñez, 1990). Se seleccionan los componentes cuyos valores produzcan un error menor a $1 \%$, de la esscala completa, y que el tiempo máximo de estabilización del circuito convertidor sea menor a 0.7 segundos, para ello se emplean las figuras 1 y 2 de Counts et al. (1982).

La función del convertidor a Vef es (Fink y Beaty, 1990):

$\operatorname{Vef}($ c.d. $)=\sqrt{\frac{1}{T} \int(\operatorname{Ve}(t) * \operatorname{Ve}(t)) d t}$

donde:

$T=$ periodo de evaluación (segundos)
En la figura 4, se presenta el circuito que convierte el $\mathrm{Ve}(t)$ en uno de valor eficaz verdadero Vef. Para ello, uti-

a) alcance del Ve(t): de $50 \mathrm{mVp}$ a $3 \mathrm{Vp}$

c) alcance del FC (Vs): de 1 a 10 (voltios)

d) error en linealidad menor a $2 \%$ de la escala completa. Vett en uno de valor eficaz verdadero Vef, Para ello, uti- 
$\operatorname{Ve}(t) \quad=\quad$ voltaje de la señal de entrada (voltios) Vef (c.d.) = voltaje eficaz (voltios).

En la figura 5 se presenta el circuito detector/retenedor del nivel de pico máximo del Ve $(t)$ construido con el amplificador operacional de entradas JFET LF347N de National Semiconductor (1984). Se diseña e implementa para obtener continuamente el nivel del pico máximo positivo (Stout y Kaufman, 1976; Coughlin y Driscoll, 1982) y se incorporan los circuitos para desacoplar la entrada (amplificador U2D/12) y reforzar la salida. En particular, en ésta última, se coloca un atenuador por 10 (amplificador U2A/1) necesario para acoplar al circuito divisor cuya función transferente así lo requiere (por ejemplo, $\left.\mathrm{Vs}=10^{*} \mathrm{Vp} / \mathrm{Vef}\right)$.

Los componentes que lo integran se eligen considerando las características y especificaciones de diseño propuesto. Este circuito es una combinación de un rectificador de onda completa de precisión con un amplifi- cador-integrador de seguimiento y retención (Stout y Kaufman, 1976).

El circuito que se implementa para obtener el cociente entre el nivel del pico máximo y el valor eficaz del Ve $(t)$ se presenta en la figura 6. Se basa en el operador analógico multiplicador/divisor AD734AN de Ana$\log$ Devices (1985) y se diseña e implementa para tal operación; los componentes que lo acompañan se eligen para que atienda las características y especificaciones mencionadas. El voltaje de salida correspondiente al FC está dado por:

$$
\mathrm{FC}=\mathrm{Vs}(\mathrm{W}, \mathrm{Y} 2)=\frac{10 \mathrm{Vp}(\mathrm{Z} 2)}{\operatorname{Vef}(\mathrm{X} 1)}
$$

donde $\mathrm{W}, \mathrm{Y} 2$, Z2 y X1 son terminales del operador mencionado.

En la figura 7, se muestra una fotografía del circuito completo en pruebas de validación.

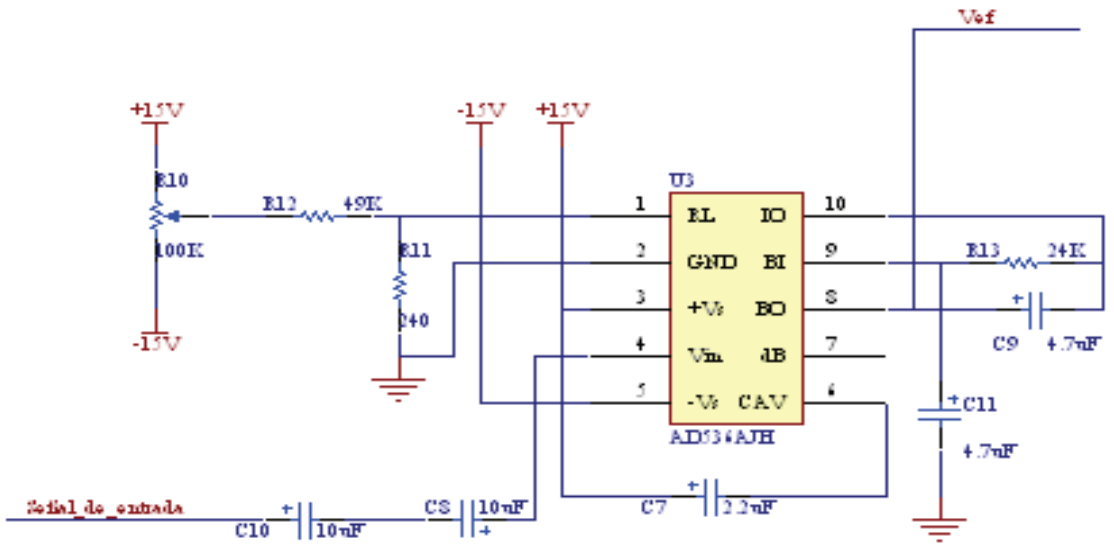

Figura 4. Circuito convertidor a valor eficaz verdadero (AD536AJH) del Ve(t)

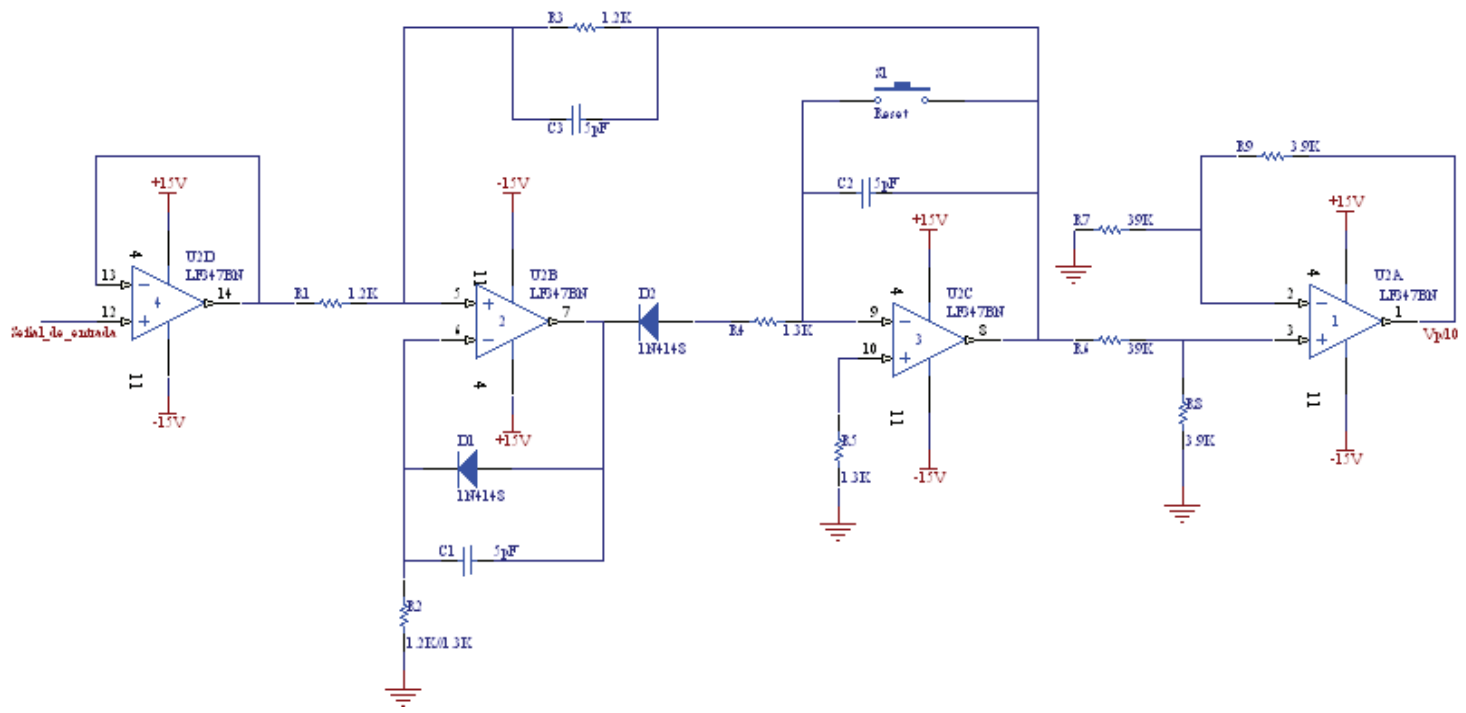

Figura 5. Circuito detector/retenedor continuo del nivel del pico máximo del Ve(t) (LF347N) 


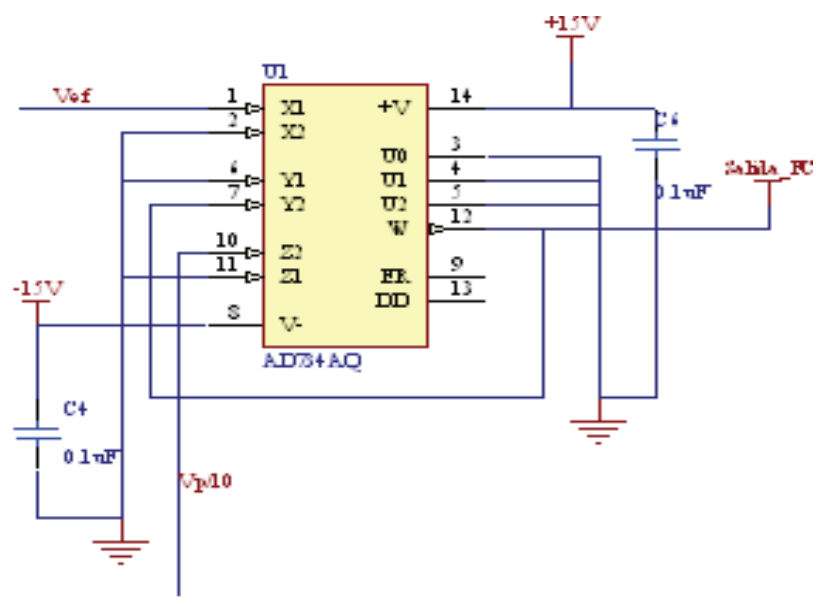

Figura 6. Circuito divisor analógico (AD734AN) que obtiene continuamente el cociente $\mathrm{Vs}$ entre el nivel del pico máximo $\mathrm{Vp}$ y el Vef de la señal $V e(t)$

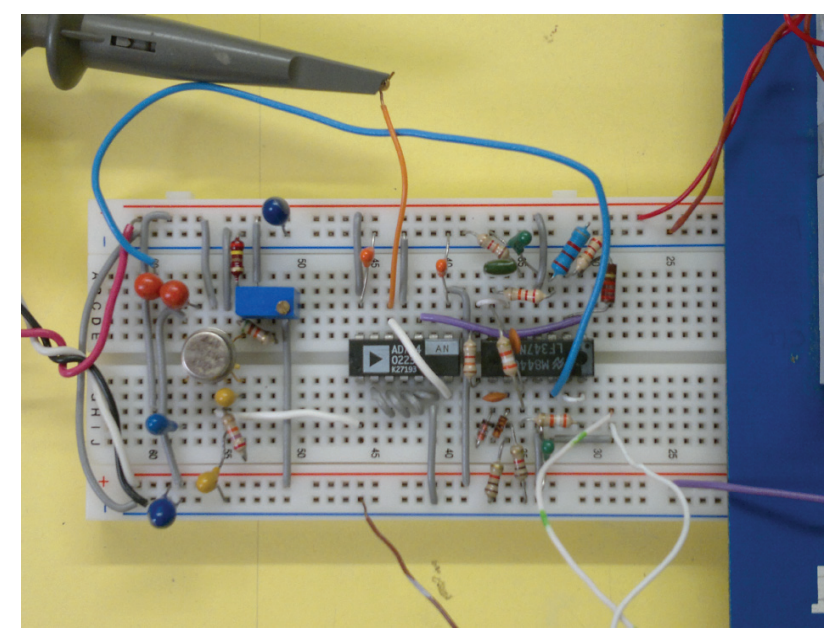

Figura 7. Tablilla de simulación mostrando el CFC en pruebas de validación

\begin{tabular}{ccccc}
\hline Ve(t), ventana W & Vp (máximo) & Valor eficaz & FC (calculado) & FC (DADISP), W \\
\hline Senoidal, W1 & 1.0006 & 0.70869 & 1.41190 & 1.433563, W3 \\
Cuadrada, W7 & 1.0160 & 1.00394 & 1.01201 & 1.008902, W9 \\
\hline
\end{tabular}

Tabla 1. Los FC de las señales adquiridas y posanalizadas con el programa DADISP

\section{Cálculo del FC utilizando los programas DADISP y LabVIEW}

\section{Versión DADISP en modo postanálisis}

El programa DADISP calcula el factor mencionado por medio de la ecuación (5), como se indica en las ventanas W2 y W3, y en W8 y W9 de la hoja de trabajo de este programa que se presenta en la figura 8, para las señales medidas senoidal y cuadrada, respectivamente. En las ventanas W4 y W6, se muestra la evolución del voltaje de valor eficaz Vef (gráfica creciente) y el traslape de éste con la propia señal y con la evolución del FC (gráfica decreciente) para cada una de las señales estudiadas.

$\mathrm{FC}($ senoidal $)=\max (\mathrm{W} 1) / \operatorname{sqrt}\left(\operatorname{partsum}\left(\mathrm{W} 1^{*} \mathrm{~W} 1\right)\right)$

Como ya se comentó, se trabaja con señales producidas por el generador de funciones Agilent 33120A (Agilent Technologies, 2000), adquiridas con el sistema automático de prueba LabVIEW y postanalizadas con el programa DADISP (Núñez, 1998). En las columnas 4 y 5, de la tabla 1, se presentan los FC calculados directamente (es decir, por el cociente entre las columnas 2 y 3 ) y por el programa DADISP para cada una de las señales (compare con los resultados de las ideales en las ventanas W2 y W6 de la figura 2).

\section{Versión LabVIEW en tiempo real}

Una de las principales ventajas que presenta el programa LabVIEW (National Instruments, 2007) es poder medir, operar y desplegar las señales o niveles de voltaje en tiempo real. Esto lo hace ideal para observar la tendencia del factor mencionado y sobre todo sus variaciones. En los incisos a) y b) de la figura 9, se presenta el programa y la señal en pantalla e indicadores numéricos que despliegan continuamente el valor del factor obtenido para el caso de la señal senoidal (compare con la señal ideal y su resultado mostrado en las ventanas W1 y W2 de la figura 2, respectivamente). Dado que ésta no está distorsionada su FC es 1.4142.

Validación del FC obtenido por el CFC y calculado por los programas mencionados

Se realiza una comparación entre el FC obtenido por el CFC y los calculados por los programas LabVIEW (en tiempo real) y DADISP (en posanálisis).

En la figura 10, en la pantalla: FC y señal, presentan la señal del Ve(t) operada (abajo) y su FC generado por el CFC (arriba). El valor instantáneo de este mismo factor se presenta en la carátula e indicador numérico: FC medido (derecha), y su tendencia se despliega en la pantalla: históricos FC y límites (derecha abajo). En los 

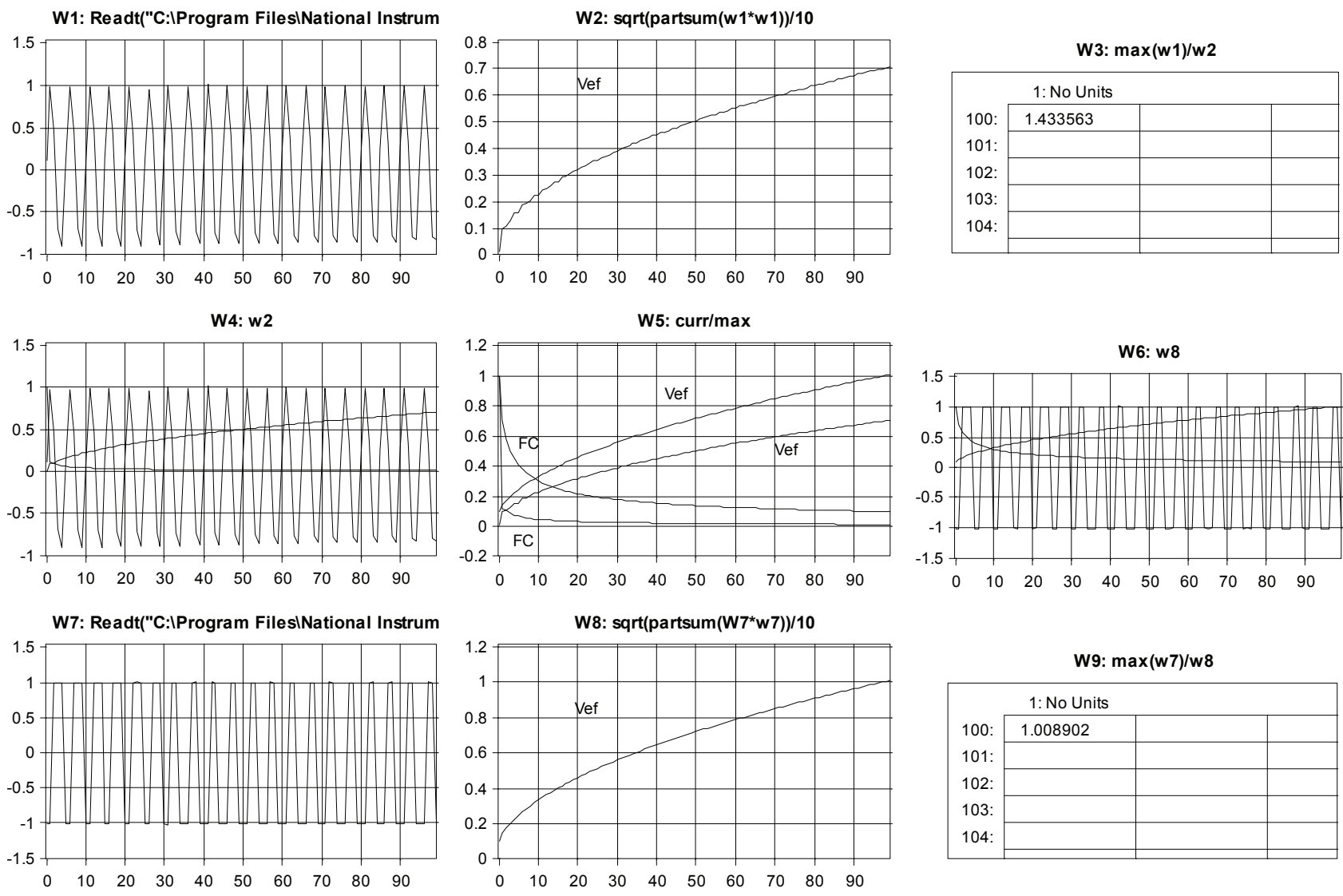

W9: $\max (w 7) / w 8$

Figura 8. Hoja de trabajo del programa DADISP; contiene las señales medidas Ve(t): W1: senoidal, W7: cuadrada, y cálculo de sus valores eficaces (W2, W8) y FC (W3, W9), respectivamente, en un esquema de posanálisis. W4 y W6, muestran las evoluciones del Vef, Ve(t) y FC para cada caso (vertical: voltios, horizontal: $\mathrm{m} / \mathrm{seg}$ )

a)

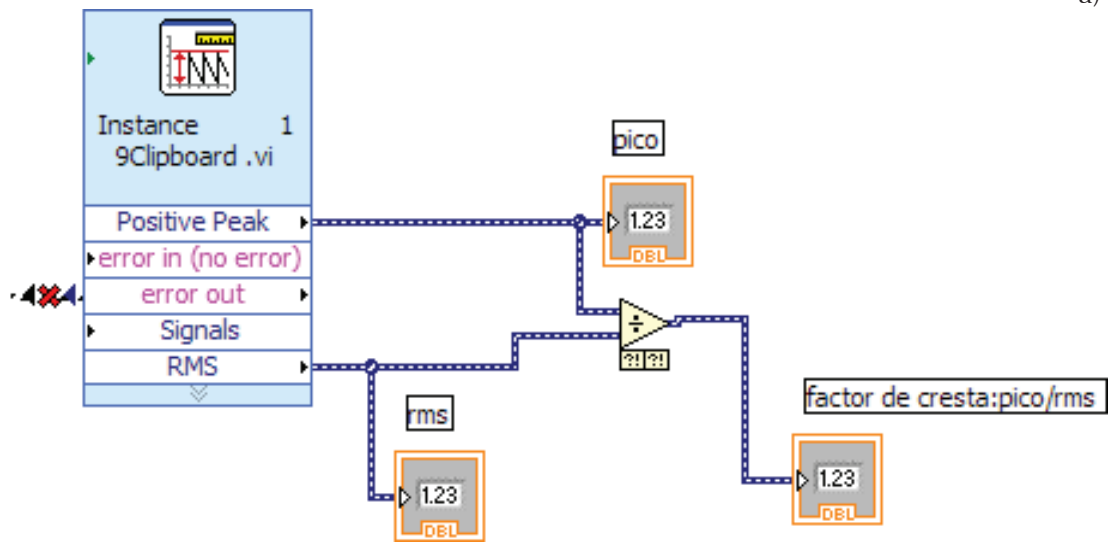

b)

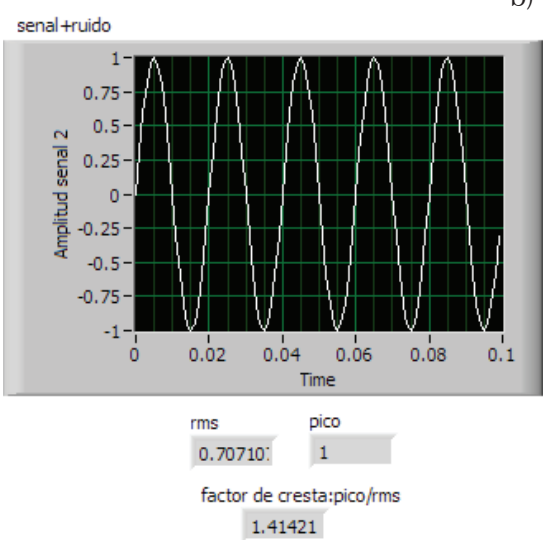

Figura 9. Programa a) y pantalla b) en tiempo real del FC calculado por el programa LabVIEW para el caso de la señal senoidal ideal (compare con la ventana W1 de la figura 2) 

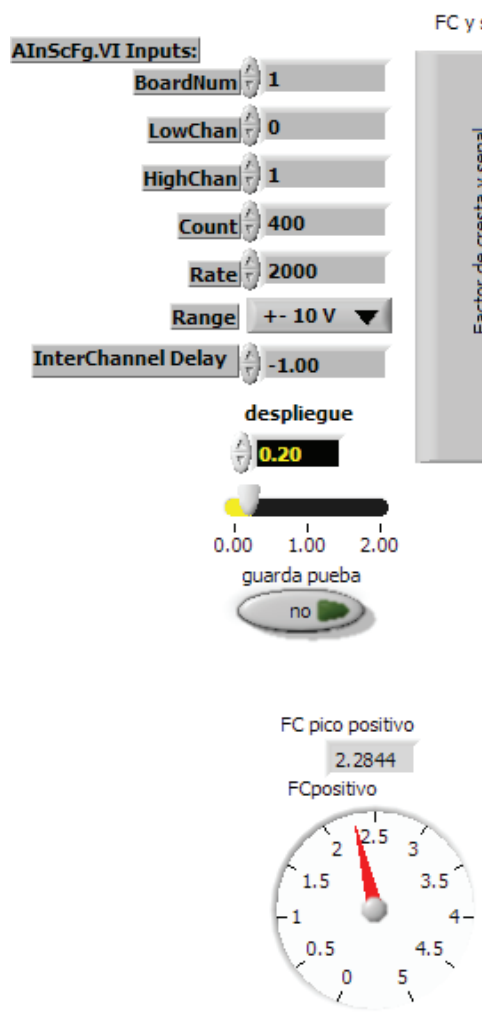
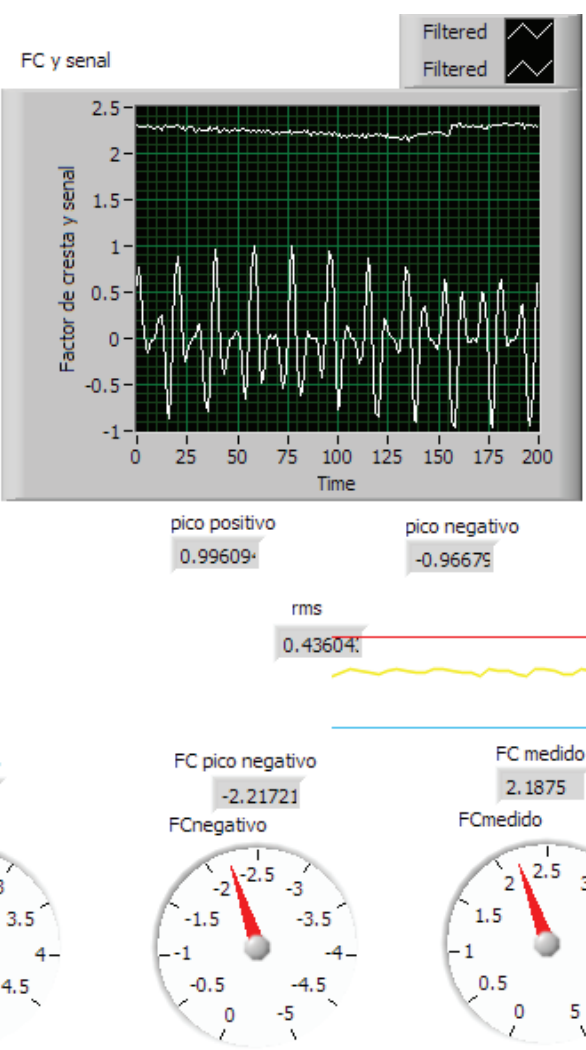

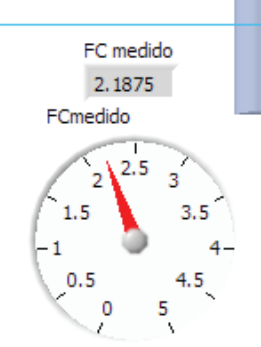

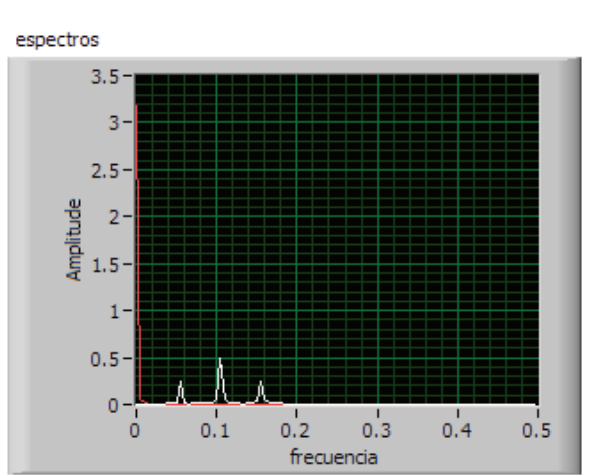

historicos FC y limites

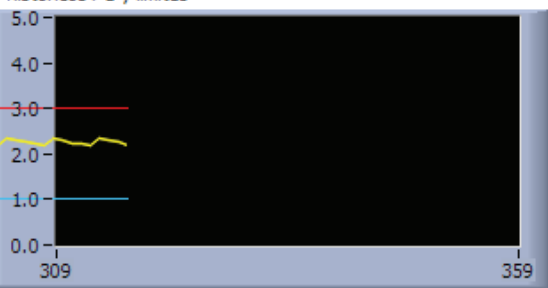

limite inferior limite superior

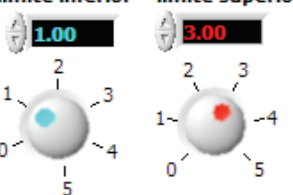

Figura 10. Pantalla e indicadores que muestran el FC calculado por el programa LabVIEW y el obtenido por el CFC y su tendencia entre límites

indicadores restantes, identificados con FC pico positivo y negativo, se presentan los valores instantáneos calculados del Ve $(t)$ por el programa LabVIEW. Para esta prueba, se escoge como $\mathrm{Ve}(t)$ una señal modulada en amplitud de $210 \mathrm{~Hz}$; en la pantalla de espectros se observa la fundamental y sus armónicas 110 y $310 \mathrm{~Hz}$ (horizontal: $\mathrm{fm} / 2$ canales $=2 \mathrm{Khz} / 2=1 \mathrm{Khz}$ a escala completa, donde: $\mathrm{fm}=$ frecuencia de muestreo).

En las ventanas W1 y W3 de la figura 11 se presenta la misma señal de prueba $\mathrm{Ve}(t)$ y su FC calculado por el programa DADISP, respectivamente. Se observa en la ventana W2 cómo evoluciona el Vef de la señal Ve $(t)$ en función del número de muestras en el tiempo (por ejemplo, durante la trama de 200 muestras y $200 \mathrm{~m} / \mathrm{s}$ de duración). En la tabla 2 se presentan los resultados de los factores obtenidos por los tres procedimientos.

\section{Aplicación del FC y su tendencia para monitorear señales y detectar el nacimiento de eventos}

Al validar y calibrar el productor (CFC) y los calculadores (programas) confiables del FC y su tendencia, se procedió a aplicarlos a 4 tipos de señales de campos di- ferentes. La selección de señales por estudiar se realiza en base a los diferentes circuitos e instrumentos desarrollados en el transcurso del tiempo en el Departamento de Electrónica. Las señales consideradas corresponden a señales de vibración mecánica, de circuitos caóticos, del sistema respiratorio y de calidad de la corriente de la red eléctrica.

Señales de vibración mecánica para diagnosticar rodamientos y rotores averiados por medio del FC

En vibración mecánica (Núñez, 1987; Félix, 1992; CSI, 1988), la tendencia del FC indica el grado de distorsión que sufre la señal sintomática producto del nacimiento y desarrollo de alguna avería en el transcurso del tiempo. Esta última puede manifestarse como una señal impulsional anormal que proviene de rodamientos con pistas rayadas, rotores desbalanceados o sueltos, reductores de velocidad con juego mecánico y todo aquello que produzca impacto, golpeteo, roce o rayadura, etcétera.

Para ilustrar lo anterior, se propone la comparación de una señal que simula una condición normal de operación con dos que simulan síntomas típicos de averías 
en rodamientos y en rotores; según estudios realizados por Bruel y Kjaer (1989a y b).

En las ventanas W4 y W6 de la figura 12, se presenta una señal de vibración típica de una chumacera del motor y su FC de 2.405809, respectivamente, en condiciones normales de operación. Mientras que en las ventanas W1 y W3, se muestra la misma señal pero ahora representa síntomas de rodamiento dañado en la chumacera del motor y con un FC de 3.976277, respectivamente. En el mismo sentido, en las ventanas W7 y W9 se presenta la misma señal, pero ahora corresponden a un síntoma de rotor del motor desbalanceado y con un FC de 1.83337, respectivamente. Como puede observarse, el FC de la señal de la chumacera de la máquina, operando normalmente, es 2.40589 y sufre una tendencia creciente o decreciente dependiendo del síntoma de rodamientos averiados o de rotor desbalanceado. Toda la generación y análisis se realiza utilizando el programa DADISP (2002).

Por otro lado, en la figura 13 se presentan los resultados del cálculo y despliegue en tiempo real del FC realizados por el programa LabVIEW. Para ello, se utiliza la pantalla: señal+ruido (centro), en la cual se muestra la señal de desbalance del rotor del motor (compare con la de la ventana W7 en la figura 12) y los indicadores numéricos (parte baja), en los que se presenta el FC (compare con el de la ventana W9 en la figura 12). De la misma manera, en la pantalla: señal recuperada (derecha), se presenta la señal de rodamientos averiados (compare con la de la ventana W1 en la figura 12) y su FC (compare con el de la ventana W3 en la figura 12) en los indicadores numéricos (arriba). La tendencia de ambos factores, obtenida por los dos programas utilizados, coincide con la esperada y publicada en la bibliografía especializada sobre el tema (Bruel y Kjaer, 1989a).

Señal caótica proveniente del circuito de Chua y su caotización por medio del FC

Para este estudio se considera la señal caótica $V \times 1$ proveniente del circuito de Chua (Núñez, 2008). Al variarse uno de los parámetros del circuito, en particular el representado por el resistor R8 (por ejemplo, se varía alrededor de los 1600 ohmios para mantener el comportamiento caótico) del diagrama eléctrico de la figura 14, la dinámica caótica o caotización también varía y por ende el factor mencionado.

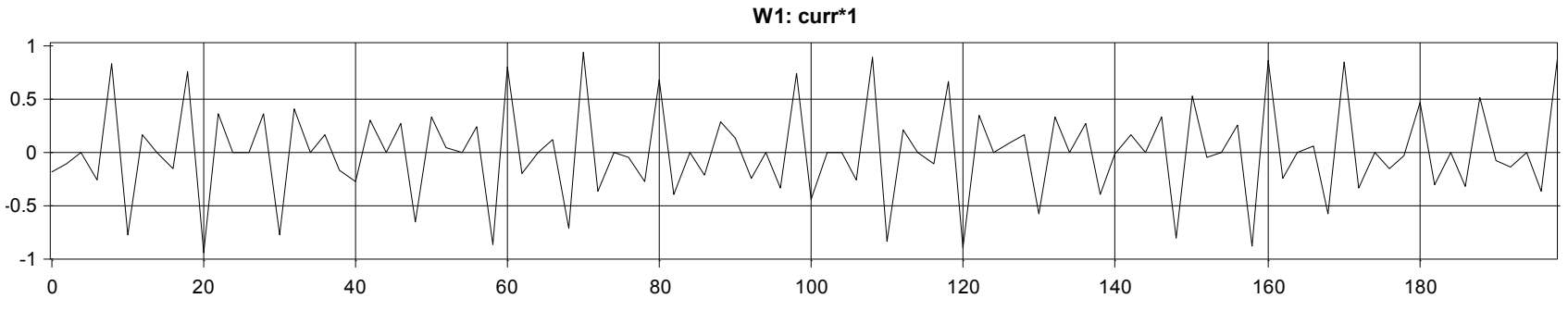

W2: $\operatorname{sqrt}($ partsum $(\mathrm{W} 1 * \mathrm{~W} 1)) / 10$

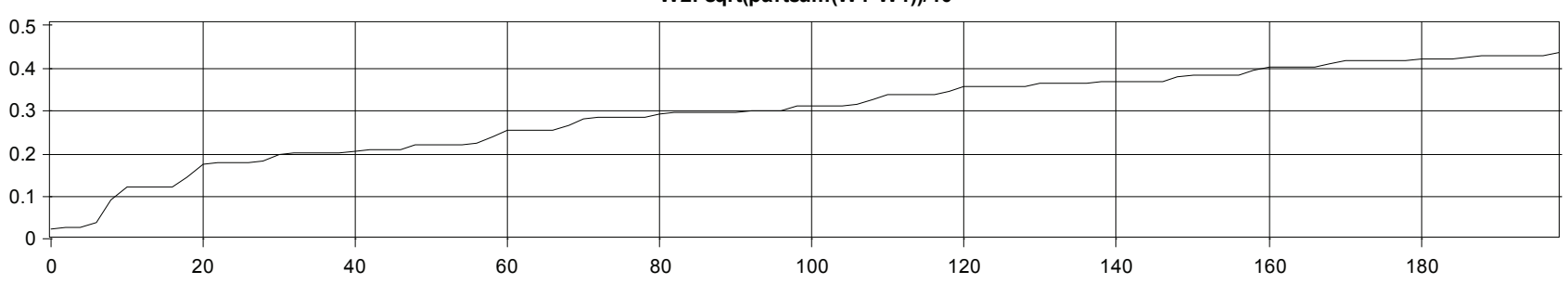

W3: $\max (\mathrm{W} 1) / \mathrm{W} 2$

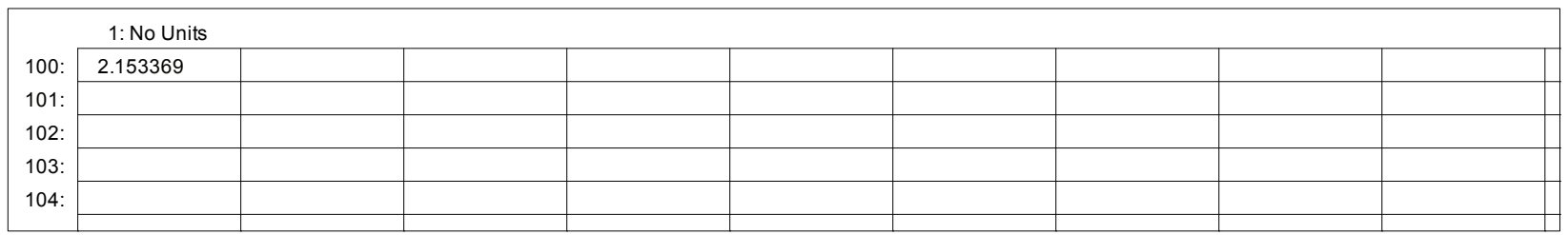

Figura 11. Resultados semejantes a los de la figura 10 (W1: señal Ve(t) y W3: FC) pero ahora con el programa DADISP (vertical: voltaje, horizontal: $\mathrm{m} / \mathrm{seg}$ ) 
Tabla 2. Comparación entre los FC: obtenido (CFC).vs. calculados (programas)

\begin{tabular}{cccc}
\hline Obtenido/calculado & CFC & LabVIEW & DADISP \\
\hline FC (voltios de c.d.) & 2.18150 & 2.2844 & 2.153369 \\
\hline
\end{tabular}
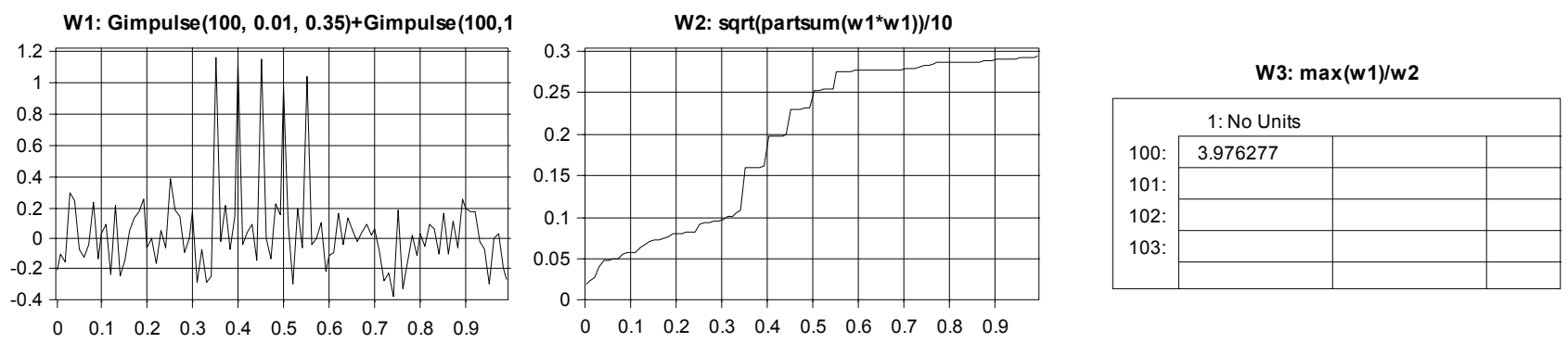

W4: $0.1^{*} \operatorname{gsin}(100,1 / 100,5)+0.3^{*}$ grandom $(100,1 /$
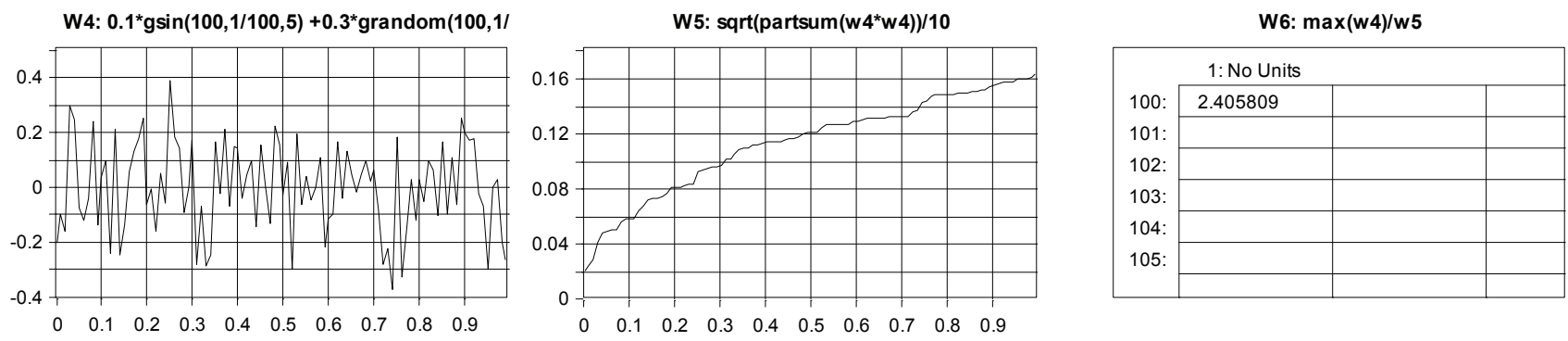

W7: $w 4+g \sin (100,1 / 100,5)$
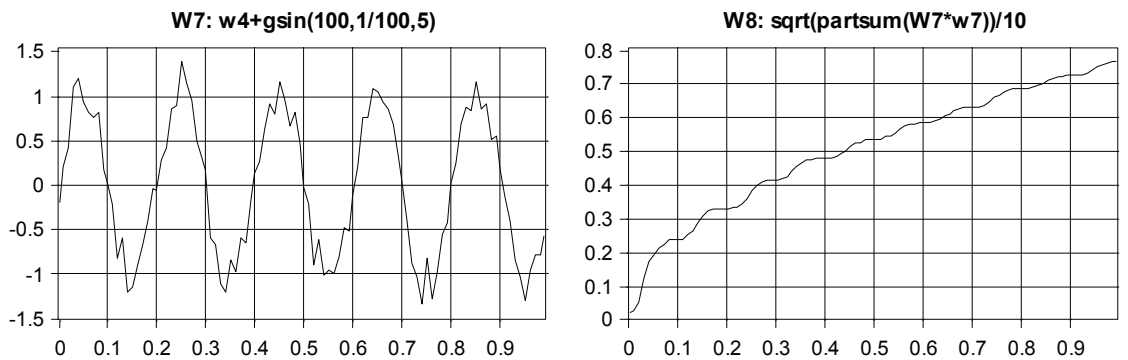

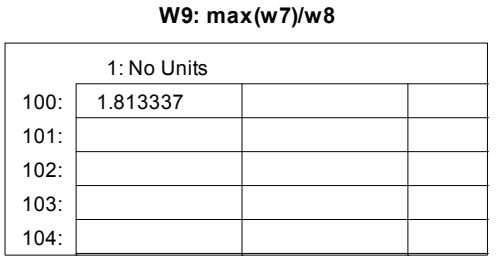

Figura 12. Señales de vibración mecánica que representan: rodamientos averiados (W1), desbalance excesivo del rotor del motor (W7) y condición normal de operación (W4), y sus FC correspondientes (W3, W6 y W9) (vertical: voltios, horizontal: m/s)

En la figura 15, se utiliza el programa DADISP para adquirir y posanalizar las dinámicas caóticas de las señales presentadas en las ventanas W1, W4 y W7 que corresponden a los valores del parámetro R8 de 1670 ohmios, de 1550 ohmios y de 1620 ohmios, respectivamente, y para las cuales se calculan sus FC que aparecen en las ventanas W3, W6 y W9 y cuyos valores son: $1.236663,1.190571$ y 1.231197 , respectivamente. Observando la dinámica caótica de las señales generadas con diferentes parámetros y sus FC obtenidos, se puede deducir que: a mayor dinámica caótica, o caosidad (Núñez, 2009), corresponde una mayor distorsión y por ende la tendencia del FC también aumenta.
Señales biomédicas representando deficiencias respiratorias comunes detectadas por el FC

Dadas las características funcionales del factor mencionado y de su tendencia, se propone que puede utilizarse en el monitoreo (es decir, en la auscultación continua) de señales respiratorias con la finalidad de identificar síntomas de deficiencias nacientes o estertores comunes (Charbonneau et al., 2000; Díaz, 2008; Quezada, 2011). En la figura 16, se presenta el diagrama funcional del IAPSER: instrumento para la adquisición y preprocesamiento de señales respiratorias desarrollado por Quezada (2011) con el cual se adquirieron las señales respi- 

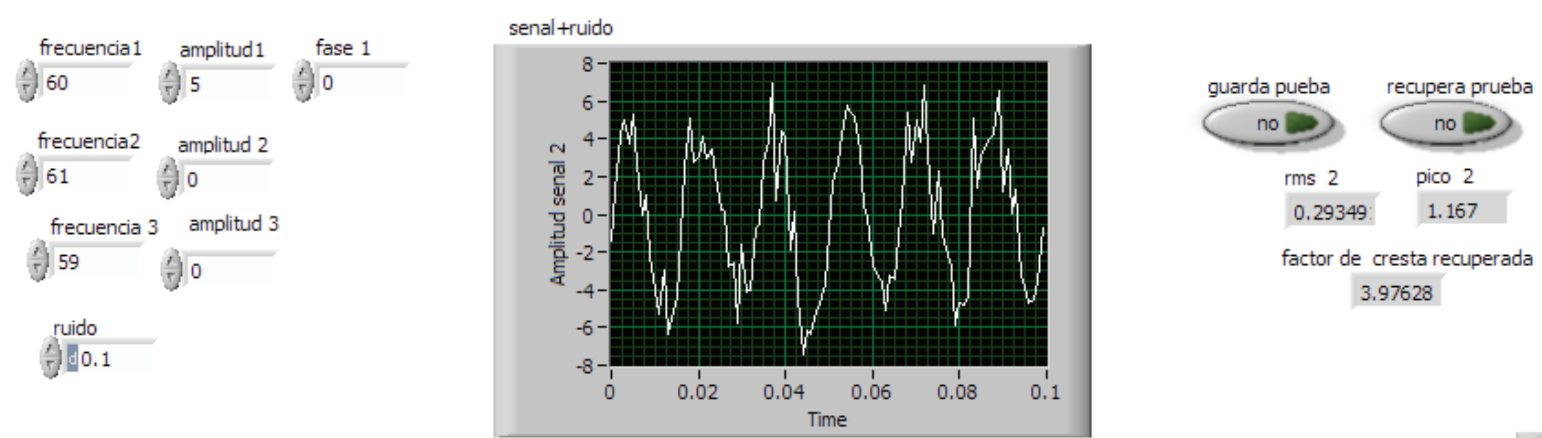

factor de cresta recuperada 3.97628
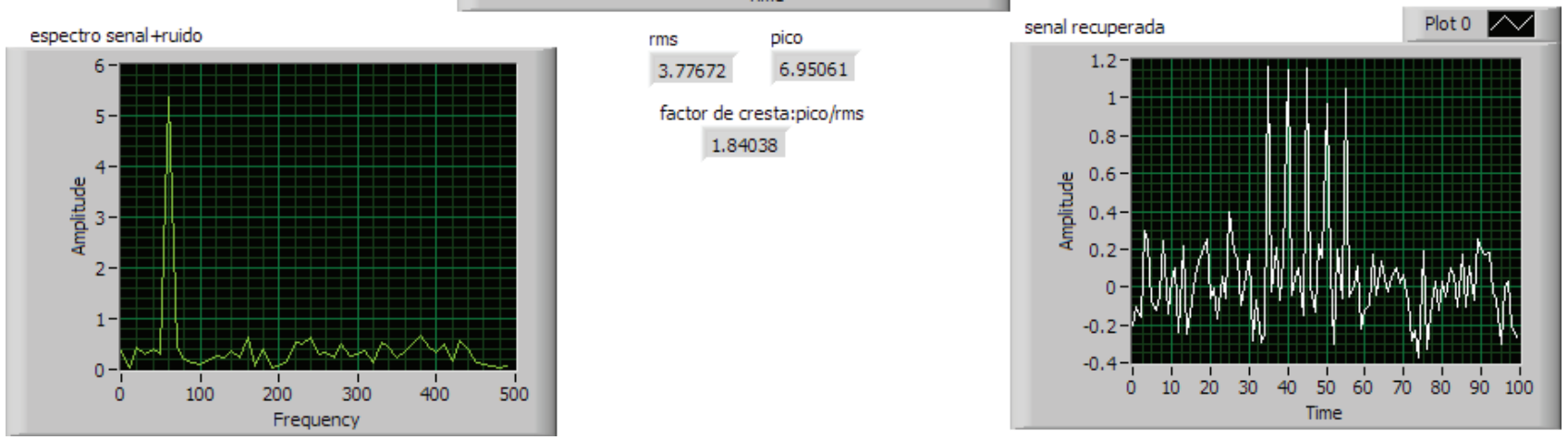

Figura 13. Señales anormales de vibración mecánica y sus FC: a) rodamientos averiados (pantalla derecha: señal recuperada) con un FC de 3.97628 y b) desbalance excesivo del rotor (pantalla central: señal+ruido) con un FC de 1.84038

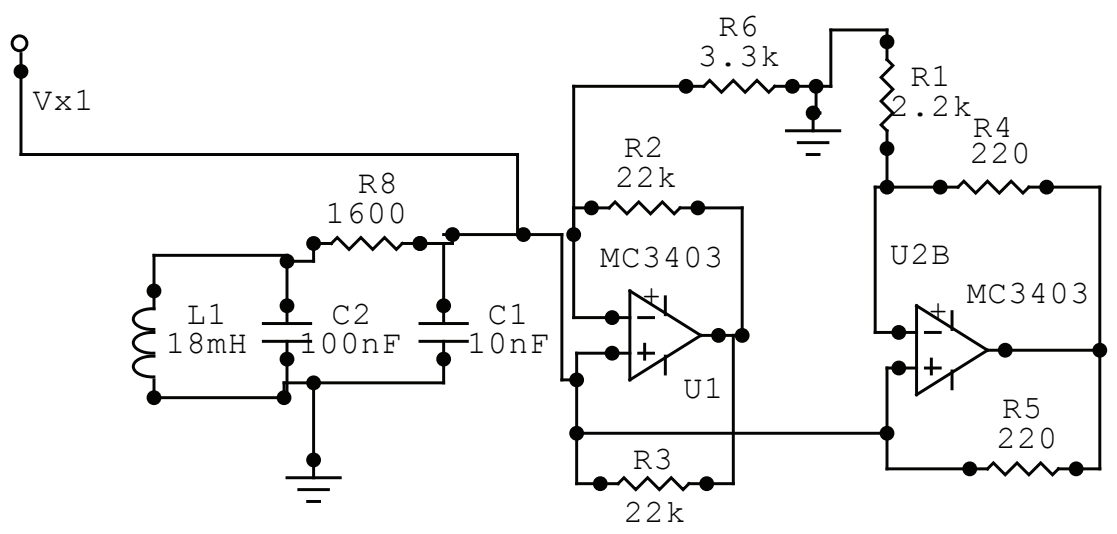

Figura 14. El circuito de Chua y la señal caótica $\vee \times 1$ estudiada ratorias de un adulto sano mostradas en la figura $17 \mathrm{y}$ que corresponden a tres ciclos respiratorios durante 10 segundos.

En las ventanas W1 y W7 de la figura 18, se presenta la señal respiratoria del adulto sano mencionado y otra de uno con deficiencias nacientes o estertores (como tos fuerte), respectivamente. Se calculan ambos FC con los niveles máximos que, para este caso, corresponden a los pulsos negativos. Se comparan y se observa una diferencia producto de la magnitud del estertor del adulto tosiendo.

Los resultados indican que para el adulto sano se tiene un valor del factor de -3.251153 y para el adulto tosiendo de -5.145114. En vista de esto, la tendencia del FC para un adulto sano debe mantenerse con valores igual o menores al -3.3, mientras que valores mayores indican el nacimiento y desarrollo de estertores $\mathrm{u}$ otras deficiencias importantes.

Evaluación de la distorsión de la corriente ante cargas no-lineales utilizando el FC

El perfil de la forma de onda de corriente nos indica la calidad de la potencia consumida por una carga en general, cuando ésta es no-lineal el perfil se distorsiona (Cáceres, 2008; Fink y Beaty, 1990; Early et al., 1989), lo 

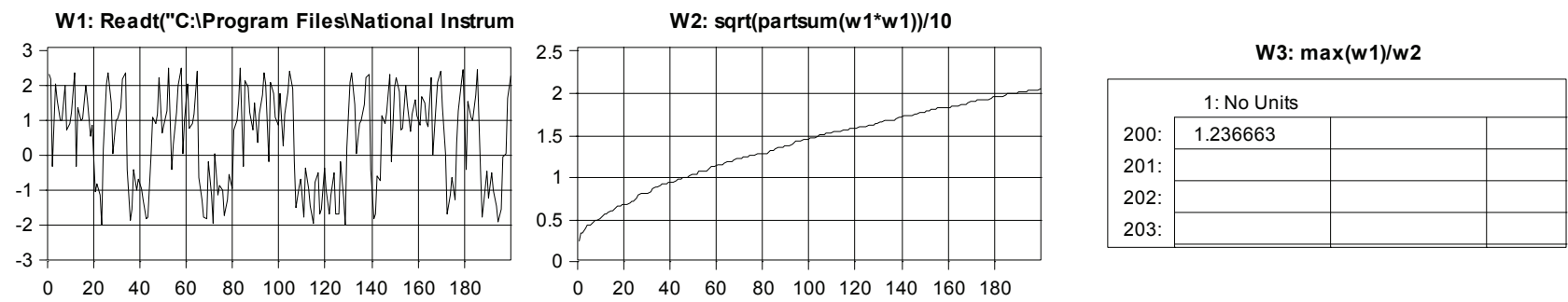

W4: Readt("C:IProgram FilesiNational Instrum
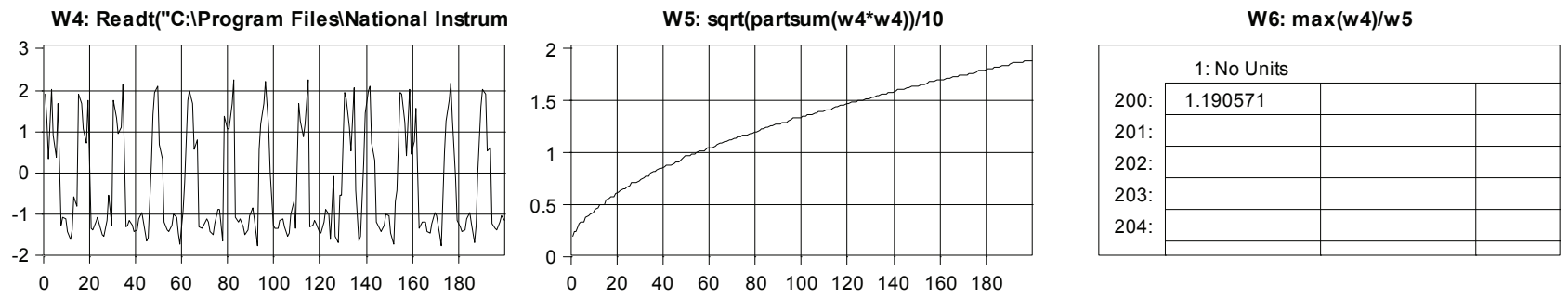

W7: Readt("C:IProgram FilesINational Instrum
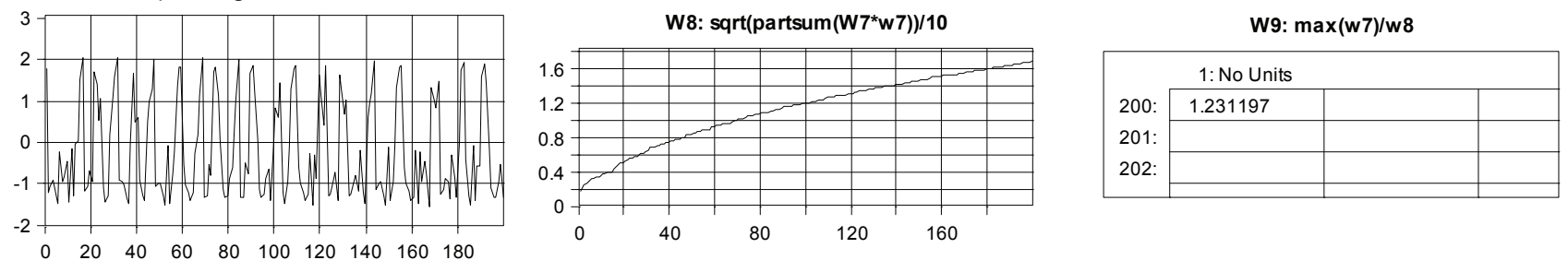

Figura 15. Señales caóticas $\vee \times 1$ del circuito Chua, generadas con parámetros diferentes y cálculo de sus FC asociados utilizando el programa DADISP. Entre más caotización mayor distorsión y la tendencia del FC también aumenta (vertical: voltios, horizontal: m/s)

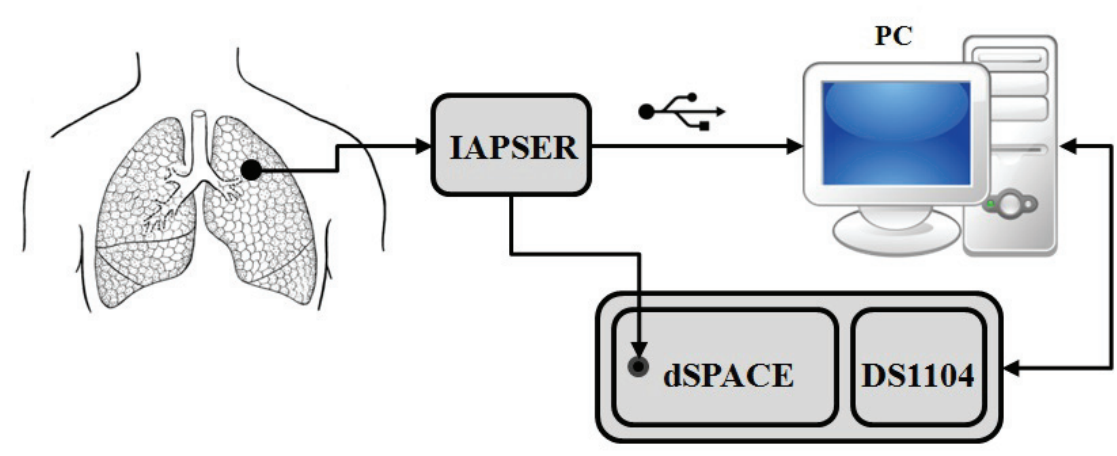

Tarjeta de adquisición
Figura 16. IAPSER: adquisidor/ preprocesador de señales respiratorias (Quezada, 2011) que acarrea que al medir la corriente con un instrumento común, esta medición no sea verdadera y por ello se necesita utilizar algún analizador de parámetros eléctricos (como el Fluke 41B), con todo lo que esto implica. La deformación del perfil mencionado la provocan generalmente componentes no-lineales e inductivos como: balastras electrónicas, controladores (con tiristores) de motores de c.a., fuentes conmutadas, entre otros. Como ya se sabe de Fluke (1990), entre más se distorsione la corriente en la carga resulta más grande su FC como se muestra en las figuras 21 y 22. A final de cuentas este factor se puede interpretar como el grado de distorsión que sufre la corriente e indica la necesidad de hacer algo para compensarla. Por ejemplo, corregir el factor de potencia por medio de capacitores o incorporando resistores en paralelo con la carga para disminuir la distorsión inductiva, entre otras acciones. Estas correcciones redundarían en dar más confiabilidad a ciertos equipos, por ejemplo: las balastras electrónicas de lámparas ahorradoras de energía que presentan FC 
DOI: https://doi.org/10.1016/S1405-7743(15)30007-X

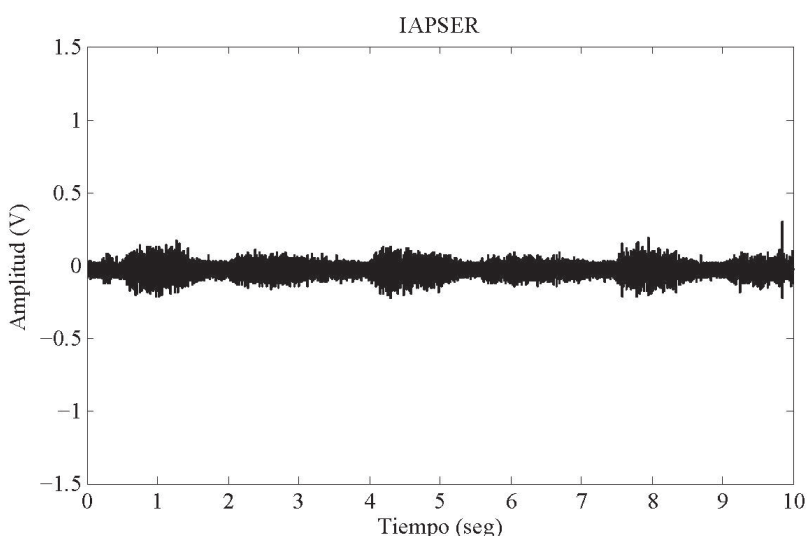

Figura 17. Adquisición de los sonidos respiratorios con el IAPSER (Quezada, 2011) correspondientes a tres ciclos respiratorios (inspiración-espiración) de un adulto sano durante 10 segundos

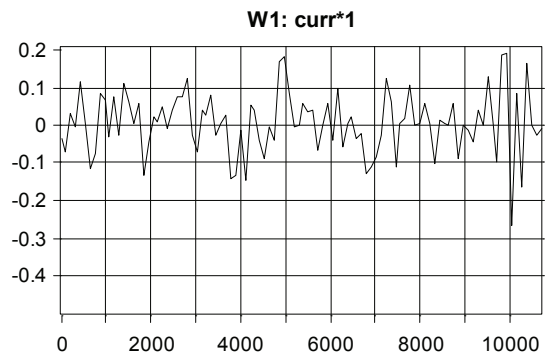

W4: Readt("C:IDocuments and SettingsIFam.

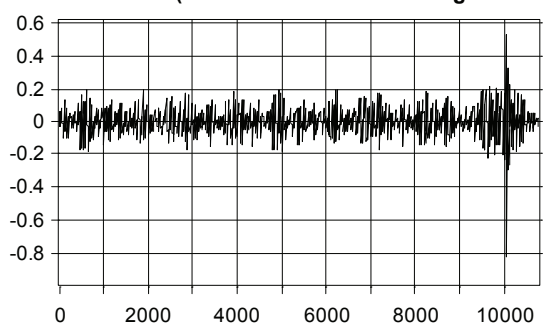

W7: decimate(w4,108)

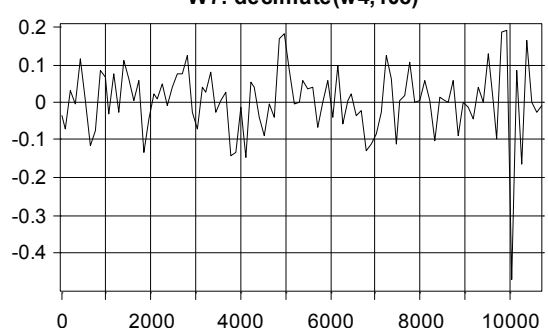

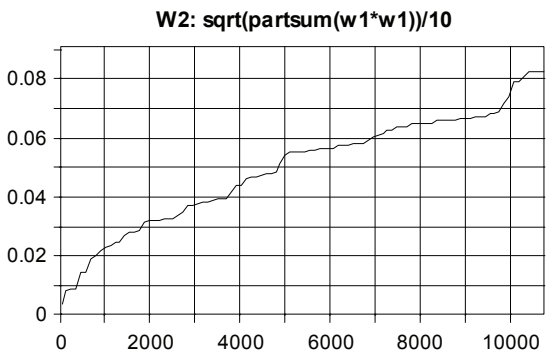

W5: sqrt(partsum $\left.\left(w 4^{*} w 4\right)\right) / 10$

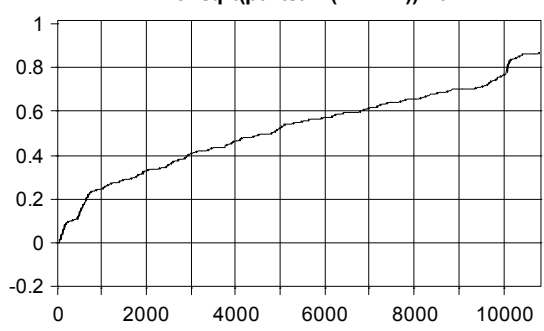

W8: sqrt(partsum $\left(W 7^{\star}\right.$ w7) $) / 10$

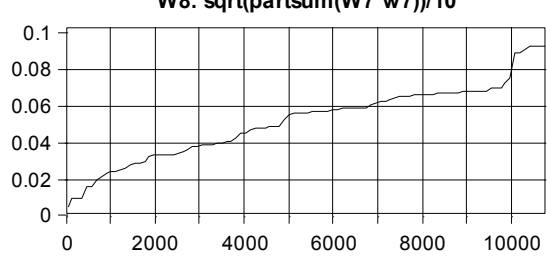

W3: $\min (w 1) / w 2$

\begin{tabular}{|l|l|l|l|}
\hline \multicolumn{1}{|l}{ 1: No Units } & \multicolumn{1}{l|}{ 100: } \\
\cline { 2 - 4 } 101: & -3.251153 & & \\
\cline { 3 - 4 } 102: & & & \\
\cline { 2 - 4 } 103: & & & \\
\cline { 2 - 4 } & & & \\
\hline
\end{tabular}

W6: $\max (w 4) / w 5$

\begin{tabular}{|l|l|l|l|}
\hline \multicolumn{3}{l}{ 1: No Units } & \\
\cline { 2 - 4 } 10784: & 0.609586 & & \\
\cline { 2 - 4 } 10785: & & & \\
\cline { 2 - 4 } 10786: & & & \\
10787: & & & \\
\cline { 2 - 4 } 10788: & & & \\
10789: & & & \\
\cline { 2 - 4 } & & & \\
\hline
\end{tabular}

W9: $\min (w 7) / w 8$

\begin{tabular}{|l|l|l|l|}
\hline \multicolumn{3}{|l}{ 1: No Units } & \\
100: & -5.145114 & & \\
\cline { 2 - 4 } 101: & & & \\
\cline { 2 - 4 } 102: & & & \\
\cline { 2 - 4 } & & & \\
\hline
\end{tabular}

Figura 18. Señales respiratorias de adulto adquiridas y posanalizadas con el DADISP, correspondientes a dos síntomas diferentes como lo indican los FC en las ventanas W3 (adulto sano) y W9 (adulto con deficiencia respiratoria o estertor) (vertical: voltios, horizontal: m/s)

grandes (Cáceres, 2008) e incorporan muchas armónicas a la línea de alimentación que se conducen y radian campos magnéticos que pueden interferir circuitos y equipos electrónicos sensibles (Ott, 1998); también estas armónicas pueden sobrecalentar secundarios de transformadores, estatores de motores, etcétera.

Como ya se dijo, el factor mencionado indica el grado de distorsión de la forma de onda de corriente; cuan- to más distorsionada esté, mayor será el valor de este factor. Normalmente un valor de 3 es más que suficiente para lograr mediciones aceptables en el sector eléctrico (Fluke, 1990; Early et al., 1989), esto siempre y cuando se considere un ancho de banda de $1 \mathrm{Khz}$.

Para el estudio en particular, las mediciones de corriente se realizan con un instrumento desarrollado, llamado: "Watthorímetro K0" (Núñez, 2010), el cual 
mide la potencia instantánea y la energía utilizando sólo un transformador toroidal de corriente.

El medidor mencionado está constituido por un transformador toroidal de corriente, un amplificador diferencial y un convertidor de valor eficaz verdadero (Kitchin y Counts, 1983; Analog Devices, 1983). En la figura 19 , se muestra a cuadros la sección mencionada y en la figura 20, la maqueta de este instrumento en operación. Como puede apreciarse en la fotografía de la figura 20, el circuito incorpora algunos contadores/ visualizadores para medir y desplegar el valor de la potencia instantánea y de la energía; si la carga es pasiva la medición es bastante precisa, pero si se hace reactiva, la carga se pierde. Por lo tanto, si se conoce el FC, se puede saber qué esperar de la señal medida; si viene distorsionada y registra un FC de 3 o menos es posible medirla aceptablemente con un amperímetro común pero si es mayor se debe utilizar un medidor de distorsión armónica total.

En la figura 21, se presentan las señales de corriente alterna instantánea en la carga y su correspondiente FC, para tres tipos de carga: a) pasiva de 25 Watts (W1), b) pasiva 60 Watts e inductiva de 13 Watts (W4) y c) pasiva 25 Watts e inductiva de 23 Watts (W7) para $120 \mathrm{Vca}, \mathrm{y}$ sus FC son: 1.265380, 1.517507 y 3.109304, respectivamente.

Se observa que la más distorsionada es la c), ya que presenta el FC mayor y resulta ser la combinación más inductiva. Las mediciones y el posanálisis se realizan con el programa DADISP (2002).

Por otro lado, en la figura 22 se presentan los resultados del cálculo y despliegue en tiempo real del FC con el programa LabVIEW. Para ello, se utiliza la panta1la: FC y señal (centro), en la cual se muestra la señal de corriente producida por una carga resistiva de 25 Watts y una lámpara ahorradora de 13 Watts (compare esta señal con la de la ventana W4 de la figura 21) y los indicadores numéricos (parte baja), en los cuales se presenta el FC (compare con el de la ventana W6 de la figura 21) y en la carátula: FC medido, se presenta el valor instantáneo medido del CFC y se grafica su tendencia en la pantalla: históricos FC y límites.

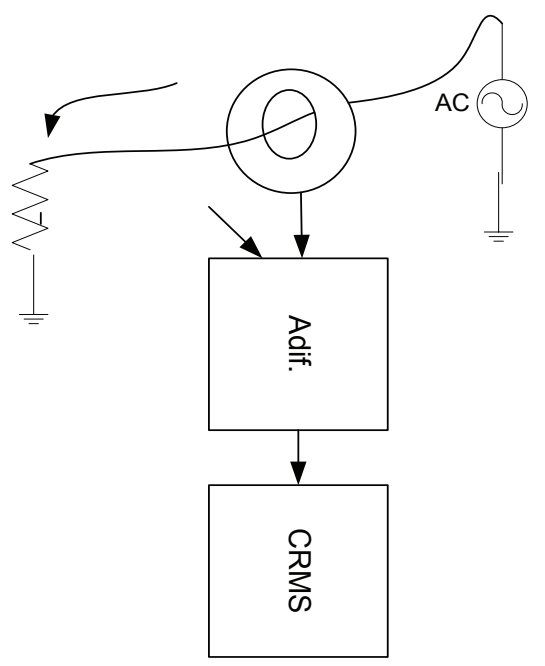

Figura 19. Bosquejo de la medición de corriente de $120 \mathrm{Vca}$ a $60 \mathrm{~Hz}$ utilizando el "Watthorímetro K0", con un transformador toroidal de corriente de 20 Amperios como sensor

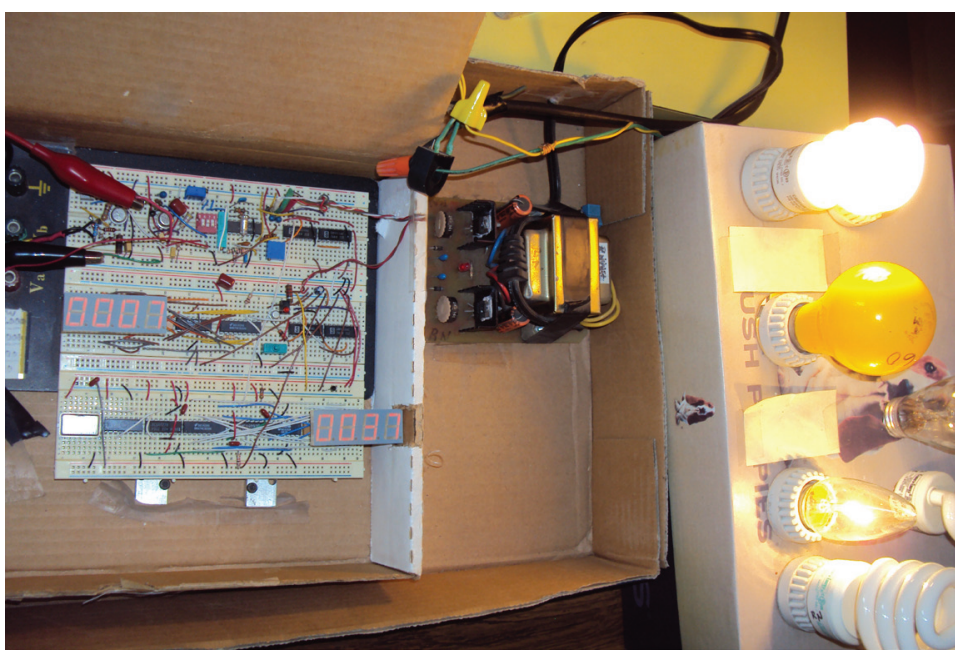

Figura 20. Maqueta del "Watthorímetro K0" en operación, mostrando el transformador de corriente (arriba-centro) y la carga combinada: pasiva de 25 Watts e inductiva de 23 Watts 
W1: Readt("C:IProgram FilesINational Instru

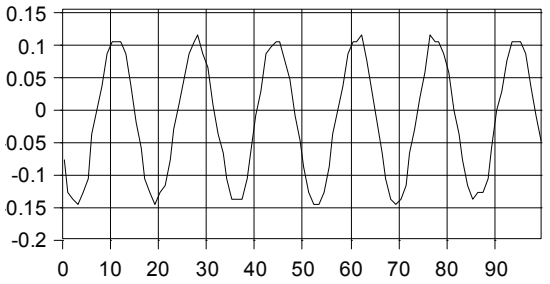

W4: Readt("C:IProgram FilesINational Instru

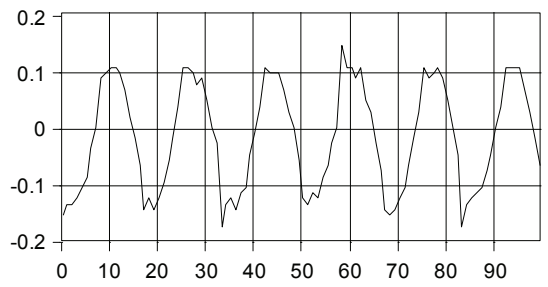

W7: Readt("C:IProgram FilesiNational Instru

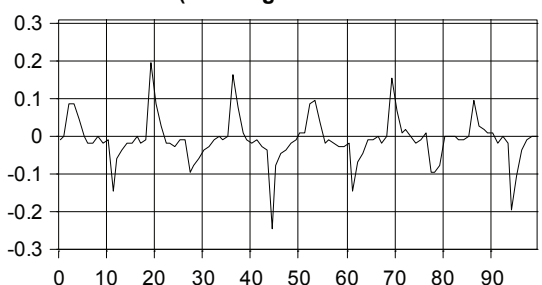

W2: $\operatorname{sqrt}($ partsum(w1*w1))/10

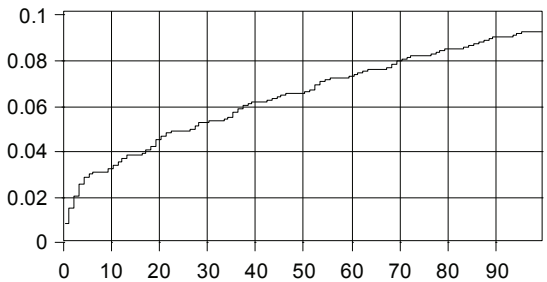

W5: sqrt(partsum(w4*w4))/10

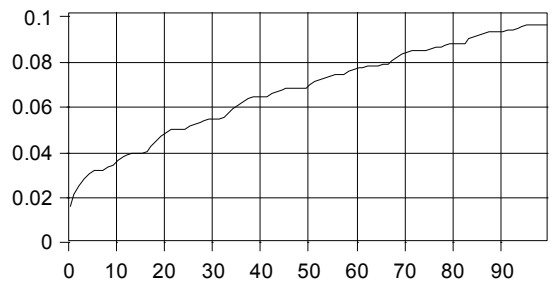

W8: $\operatorname{sqrt}\left(\right.$ partsum $\left.\left(w 7^{*} w 7\right)\right) / 10$

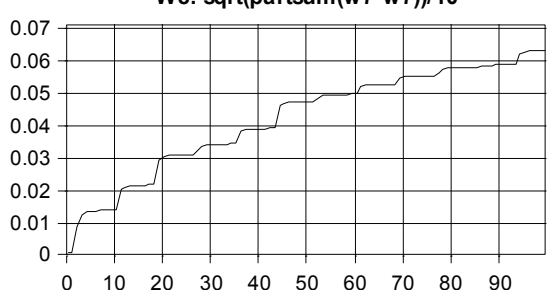

W3: $\max (\mathrm{W} 1) / \mathrm{w} 2$

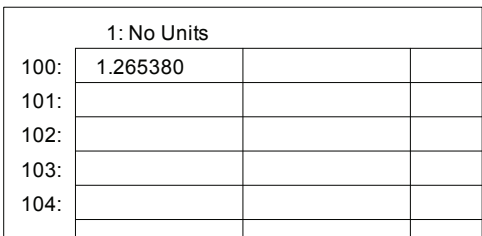

W6: $\max (\mathrm{W} 4) / w 5$

\begin{tabular}{|l|l|l|l|}
\hline \multicolumn{3}{|l}{$1:$ No Units } & \\
100: & 1.517507 & & \\
101: & & & \\
\cline { 3 - 4 } $102:$ & & & \\
\cline { 3 - 4 } $103:$ & & & \\
\cline { 2 - 4 } $104:$ & & & \\
\cline { 2 - 4 } & & & \\
\hline
\end{tabular}

W9: $\max (\mathrm{W} 7) / \mathrm{w} 8$

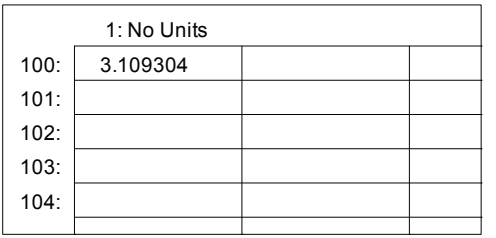

Figura 21. Medición de la corriente alterna en una carga resistiva de 25 Watts (W1) y en una lámpara con balastra electrónica inductiva de 13 Watts (W4) y 23 Watts (W7), utilizando el programa, DADISP. Se muestran sus correspondientes FC manifestando el grado de distorsión impuesto por las cargas (vertical: voltios, horizontal: $\mathrm{m} / \mathrm{s}$ )

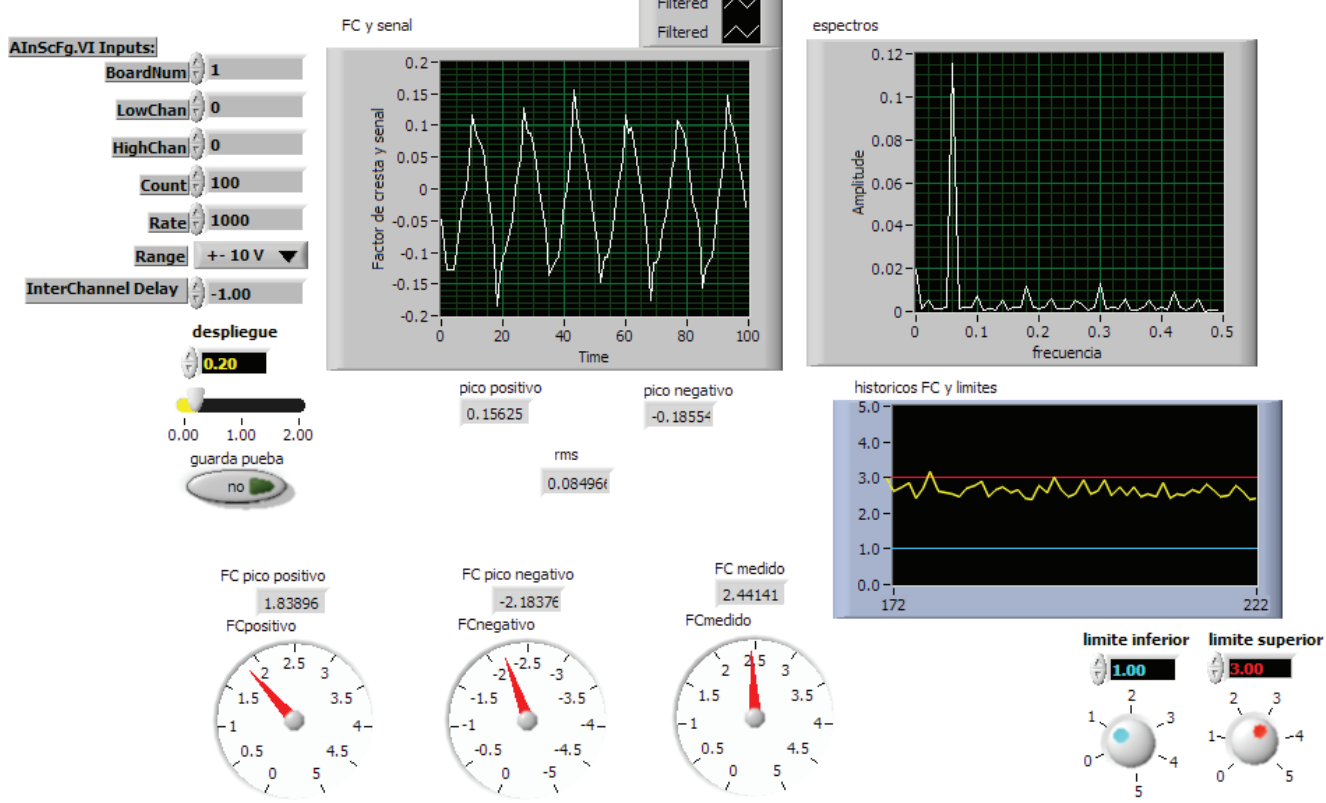

Figura 22. Medición en tiempo real de la corriente en una carga combinada: resistiva de 25 Watts e inductiva de 13 Watts para 120 Vca utilizando el programa LabVIEW. Se muestra un FC medido de 2.44141 en la carátula de FC medido y su tendencia en la pantalla históricos FC y límites 


\section{Análisis de resultados y recomendaciones generales}

Análisis de resultados de la sección de definición del FC

1. Los resultados de la evaluación del CFC implementado son prometedores, ya que al comparar sus mediciones con los valores esperados de linealidad las diferencias fueron menores a $2 \%$, respecto a la escala completa, esto es, (Vsideal-Vsmedido)/Vsideal ${ }^{*}$ 100\% (Wolf, 1973, ANSI/ISA, 1979). La obtención del voltaje del FC cumplió con las características y especificaciones propuestas para los alcances del $\mathrm{Ve}(t)$ de: a) voltaje: $50 \mathrm{mVp}$ a $3 \mathrm{Vp} \mathrm{y} \mathrm{b)} \mathrm{frecuencia:} 10 \mathrm{~Hz}$ a $1 \mathrm{KHz}$. También se logran una precisión y reproducibilidad muy aceptables, producto de aplicar algunas técnicas fundamentales para abatir el ruido eléctrico $(\mathrm{Ott}$, 1998), tanto en la señal del Ve(t) como en las diferentes etapas del circuito electrónico construido.

2. La comparación de los FC de señales medidas de referencia, calculados por el programa DADISP de la figura 8 y reportados en la tabla 1 arrojan resultados aceptables y más si se comparan con los ideales de la figura 2, se puede decir que son muy cercanos dado que los errores fueron de $-1.37 \%$ y $-0.89 \%$ para la señal senoidal y para la cuadrada, respectivamente. Las pequeñas diferencias se deben principalmente a un desbalance intrínseco en los canales del sistema de adquisición (Núñez, 1998).

3. La validación realizada al factor obtenido por el CFC y al calculado por los programas DADISP y LabVIEW fue representativa y sus cuantificaciones se muestran en la tabla 2. Como era de esperarse, también son muy cercanas, pese a que las mediciones se realizaron en forma asincrónica. El circuito y el programa LabVIEW producen resultados en tiempo real, mientras que el DADISP los produce en posanálisis, para la señal de prueba del $\mathrm{Ve}(t)$ modulada en amplitud de $210 \mathrm{~Hz}$ y $1 \mathrm{Vp}$. Se corrobora cualitativamente estos resultados con los de la tendencia del factor de la figura 10, mostrada en las pantallas de PC y señal (superior) y la de históricos FC y límites.

Análisis de resultados de la sección de aplicaciones

Habiendo validado y calibrado al productor y a los calculadores confiables del FC, se procedió a aplicarlos a 4 tipos de señales de campos diferentes: detección de averías mecánicas, medición de la caosidad, detección de deficiencias respiratorias y medición de la distorsión en la corriente eléctrica.
1. En el caso de detección temprana de averías mecánicas simuladas, se muestra claramente cómo la tendencia del factor pasa de una máquina operando normalmente con un FC de 2.405809 a una con los rodamientos averiados y un FC de 3.976277 y después a otra con un rotor desbalanceado en exceso y con un FC de 1.813337. En ese sentido, la idea es que la tendencia del factor debe mantenerse dentro de los límites especificados por el analista y la normatividad correspondiente para el tipo de máquina en particular, como lo indica claramente la figura 13.

2. En el caso de medición de la dinámica caótica o caosidad de la señal del circuito de Chua, se presenta la tendencia del factor mencionado, de un grado de caosidad nominal, generado por un parámetro en particular y produciendo un FC de 1.190571, a un aumento y disminución del mismo conforme lo hace el parámetro. El FC o grado de caosidad pasa de 1.236663 a 1.231197 para las condiciones paramétricas mencionadas. Los resultados de los factores mencionados se obtienen de las ventanas W3, W6 y W9 de la figura 15.

3. En el caso de detección temprana de deficiencias respiratorias comunes, se observa en la figura 18 como el FC del adulto normal es -3.251153 y con estertor es -5.145114 , por lo que para un factor mayor que -5.145114 se registran síntomas de estertores o disfunciones del sistema respiratorio; condición que debe reportarse instantáneamente al vigilante del paciente. Es conveniente recordar que, para este caso en particular, los resultados son de carácter preliminar y básicamente demostrativos.

4. En el caso de medición de la distorsión eléctrica, utilizando la corriente medida por el Watthorímetro $\mathrm{K} 0$ para obtener su FC, se puede observar claramente que el grado de distorsión obtenido se representa por el factor mencionado como lo indican las figuras 21 y 22, y es producto de las cargas combinadas; la más inductiva registra la mayor distorsión, -como era de esperarse- y que se presenta en la ventana W7 de la figura 21, también en la ventana W9 de la misma figura se muestra su mayor FC.

\section{Algunas recomendaciones generales}

Para detectar tempranamente eventos de trascendencia en la condición de operación, la clave es observar y analizar las variaciones de la tendencia del FC. El CFC es confiable y puede utilizarse en aplicaciones no convencionales y más si se rediseña para utilizarlo con señales de muy bajas frecuencias. 
Otro uso del FC es como elemento de retroalimentación para el control del caos en sistemas mecánicos mediante la variación de un parámetro producto de la caosidad predeterminada por el usuario.

Para mejorar la precisión en la medición de la potencia eléctrica, el Watthorímetro K0 que utiliza un transformador de corriente para medir la corriente de cargas no-lineales debe basarse en el FC para mejorar el factor de potencia. Se contempla como trabajo a mediano plazo analizar y aplicar un FC construido con el cociente de la suma de las señales máxima positiva y negativa.

\section{Conclusiones}

Se comprueba experimentalmente que la tendencia del factor de cresta FC permite obtener una idea de lo que está y sigue sucediendo con una señal periódica de información durante un tiempo predeterminado, ante la presencia de señales impulsivas, perturbadoras, generadas por eventos que pueden significar averías o deficiencias en los diferentes sistemas de los campos del conocimiento.

Se desarrolla un circuito electrónico CFC para obtener el FC y se describe el cálculo de este factor por medio de los programas DADISP y LabVIEW; se validan y corroboran los resultados de todos los factores por medio de un estudio comparativo entre ellos ( $y$ sus tendencias) y las características y especificaciones planteadas. Los resultados fueron aceptables de tal suerte que se utilizan estos procedimientos para detectar averías (simuladas) tempranas en máquinas eléctricas, identificar diferencias en la caosidad de señales medidas de circuitos caóticos, detectar deficiencias respiratorias o estertores anormales en señales medidas con desarrollos propios y, finalmente, para identificar distorsiones en la corriente eléctrica medida con el Watthorímetro K0.

Como se pudo comprobar, la obtención del factor mencionado es sencilla y por ende arroja resultados rápidamente, es decir, manifiesta tempranamente la cantidad de eventos nacientes o impactos.

Aunque, en algunos casos, es necesario tener cuidado, ya que puede alterarse debido al ruido eléctrico que se incorpora a las señales periódicas de información. Otras aplicaciones importantes y originales del FC que se proponen son: a) controlar el caos en circuitos electrónicos que agitan/mezclan procesos industriales y b) corregir el factor de potencia de cargas no-lineales e inductivas. Se contempla a mediano plazo estudiar y utilizar un FC que considere la señal máxima de pico a pico; se piensa que esto podría mejorar su detección.
Finalmente, se puede decir que la clave es observar y analizar la variación de la tendencia del FC para detectar temprano eventos de trascendencia en la condición de operación de los sistemas.

\section{Agradecimientos}

Agradecemos al CONACYT el apoyo económico para el presente, a través del proyecto 7453 , dirigido por el Dr. J. Álvarez, y al Ing. A. Zacarías por participar en la prueba y validación del CFC.

\section{Referencias}

Agilent Technologies, 33120A Function Generator, manual 90005. ed. 6, 2000, pp. 66-67.

Analog Devices, Inc. Integrated Circuit True Rms-to-Dc Converter, AD536A, AN-C502c-4, 1983.

Analog Devices, Inc. Handbook of Analog Multipliers/Dividers, AD734, 1985, 2.55-2.66, 1985.

ANSI/ISA-S51.1. Process Instrumentation Terminology, ISA standard, 1979.

Beckman Industrial, Corp. Digital Multimeters Hand Held Models, manual, 1984, 3000-940-138-C.

Bruel \& Kjaer Instruments, Inc. Digital Signal Analysis-Using Digital Filters and FFT Techniques, Technical Review, Denmark, enero 1985.

Bruel \& Kjaer Instruments, Inc. Detecting Faulty Rolling-Element Bearings, AN-BO0210-11, 1989a.

Bruel \& Kjaer Instruments, Inc. Integration Vibration Meters 2513/16, catalog, USA, 1989b, pp. 904.

Cáceres R. Factor de utilización de la potencia. IEEELATIN AMERICA TRANSACTS, volumen 6 (número 1), 2008.

Charbonneau G., Ademovic E., Cheetham B., Malmberg L., Vanderschoot J., Sovijarvi A. Basic Techniques for Respiratory Sound Analysis. Eur. Respir. Rev., volumen 10 (número 77), 2000: 625-35.

Computational Systems Incorporated (CSI), Diagnostic Features and Applications of CSI Machinery Analysers, AN-P-001, 1988, pp.1-7.

Coughlin R. y Driscoll F. Operational Amplifiers and Linear Circuits, 2a ed., P-H, Inc., 1982, pp. 131-153.

Counts L., Kitchin Ch., Jung W. Low-Cost Rms/Dc ic's ac Measurements, EDN, enero 20, 1982, pp.101-10.

DADISP. The DADISP-SE Worksheet, DSP Development, Co., Cambridge, MA, USA, 2002.

Díaz I. Desarrollo de un sistema de adquisición de sonidos respiratorios, tesis (maestría), CICESE, 2008.

Early M., Murray R. y Caloggero J. The National Electrical Code, Handbook, 1989.

Félix R. Análisis y validación espectrales de vibración en maquinaria rotatoria, tesis (maestría), CICESE, 1992. 
Fink D. y Beaty H. Standard Handbook for Electrical Engineers, 13 ed., McGraw-Hill, 1990, pp. 2-16.

Fluke Inc. Confía usted en las medidas de su multímetro? NA10047-SPA-01, USA, 1990.

Kitchin Ch. y Counts L. RMS to DC, conversion application, ANG803-30, AD, Inc., 1983.

Lancaster D. Tech Musings-Crest Factors, AN-125.1, Synergetics, Thatcher, AZ, junio 1998.

Lorenz E.N. Deterministic Nonperiodic Flow. J. Atmosphere., Sci., volumen 20, 1963: 130-41.

Márquez A. y Álvarez J. Circuito de Lorenz, Reporte técnico, DETCICESE, 1996.

National Instrument-NI, Inc. LabVIEW7 handbook, 2007.

National Semiconductor-NS, Inc. Handbook of Operational Amplifiers/Buffers, LF347 wide Bandwith Quad JFET Input Operational Amplifier, 1984, pp. 3.14-3.21.

Núñez R. Sistema monitor de vibraciones-SIMOVI, tesis (maestría), CICESE, 1987.

Núñez R. Monitor portátil de vibración para mantenimiento de maquinaria, Mundo Electrónico-203, Boixareu eds., Barcelona, España, febrero 1990, pp. 71-76.

Núñez R. The LabVIEW (Dynamic Analyzer) and Dadisp ATS's. Reporte técnico, DET-CICESE, 1998.

Núñez R. Comunicador experimental privado basado en encriptamiento caótico. Revista Mexicana de Física, FC-UNAM, volumen 52 (número 3), 2006, pp. 285-294.

Núñez R. Measurement of Chua Chaos and its Applications, JART, UNAM, volumen 6 (número 1), abril 2008.
Núñez R. Spectrum Richness as Determinant of Chaotic Synchronization. IEEELATIN AMERICA TRANSACTIONS, volumen 7 (número 5), 2009.

Núñez R. Watthorímetro K0, Reporte técnico, DET-CICESE, 2010.

Núñez R. Aplicación en instrumentación del análisis digital de señales, curso: ET631, DET-CICESE, 2011.

Ott H. Noise Reduction Techniques in Electronic Systems, 2a ed., Wiley and Sons, 1998.

Quezada I. Desarrollo de un instrumento adquisidor de señales respiratorias, tesis (maestría), CICESE, 2011.

Stout D. y Kaufman M. Handbook of Operational Amplifier Circuit Design, Mc G-H, 1976, pp. 8.1-8.3.

Wolf S. Guide to Electronic Measurements and Laboratory Practice, Prentice-Hall, Inc., 1973.

\section{Este artículo se cita:}

Citación estilo Chicago

Núñez-Pérez, Ricardo Francisco. La tendencia del factor de cresta ayuda a detectar eventos nacientes: circuito electrónico, programas y aplicaciones a señales de diversos campos. Ingeniería Investigación y Tecnología, XV, 01 (2014): 63-81.

\section{Citación estilo ISO 690}

Núñez-Pérez R.F. La tendencia del factor de cresta ayuda a detectar eventos nacientes: circuito electrónico, programas y aplicaciones a señales de diversos campos. Ingeniería Investigación y Tecnología, volumen XV (número 1), enero-marzo 2014: 63-81.

\section{Semblanza del autor}

Ricardo Francisco Núñez-Pérez. M. en C. en instrumentación electrónica (87), CICESE, México y Lic. en ingeniería electrónica (80), UABC, México con mención honorífica en ambos grados. Desde 1987 a la fecha es profesor/investigador en el Departamento de Electrónica y Telecomunicaciones del Centro de Investigación Científica y de Educación Superior de Ensenada (CICESE). Recibió el 1er. lugar al concursar con la tesis de maestría en el IV Certamen Nacional sobre diseño de Equipo Electrónico Aplicado al Sector Eléctrico, organizado por el IIE, CONACYT, SEP y la CFE (88), y el 1er. lugar y la presea en ciencias y tecnología en el IV Certamen Nacional del CREA, área electrónica (88). Recibió un reconocimiento por dirigir la tesis de la licenciatura ganadora del 1er. lugar en el VI Certamen Nacional sobre diseño de equipo electrónico aplicado al sector eléctrico, organizado por el IIE, CONACYT, SEP y CFE (90), perteneció al SNI del 88 al 93. Sus áreas de investigación e instrucción son: desarrollo de instrumentación electrónica industrial, estudios de circuitos caóticos y sus aplicaciones, utilización de los PDS en el análisis, síntesis y control de señales caóticas, biomédicas y de vibración mecánica. 